\title{
Gp78-mediated basal mitophagy promotes mitochondrial health and limits mitochondrial ROS production
}

Parsa Alan ${ }^{1 *}$, Bharat Joshi ${ }^{1 *}$, Ben Cardoen ${ }^{2 *}$, Kurt Vandevoorde ${ }^{1 *}$, Guang Gao ${ }^{1}$, Yahya Mohammadzadeh $^{1}$, Ghassan Hamarneh ${ }^{2 \#}$, and Ivan R. Nabi ${ }^{1 \#}$

${ }^{1}$ Life Sciences Institute, Department of Cellular and Physiological Sciences, University of British Columbia, Vancouver, BC V6T 1Z3, Canada

${ }^{2}$ School of Computing Science, Simon Fraser University, Burnaby, BC V5A 1S6, Canada

*,\# equal contribution

Running title: Gp78 basal mitophagy regulates ROS

§To whom correspondence should be addressed: Ivan R. Nabi, Department of Cellular and Physiological Sciences, Life Sciences Institute, University of British Columbia, 2350 Health Sciences Mall, Vancouver, BC V6T 1Z3 Canada.

Tel: +1-(604) 822-7000

E-mail: irnabi@mail.ubc.ca

Key words: Gp78 ubiquitin ligase; mitochondria; mitophagy; reactive oxygen species; GFP-mRFP tandem fluorescent-tagged LC3; spot detection; SPECHT 


\begin{abstract}
Mitochondria are major sources of cytotoxic reactive oxygen species (ROS) that contribute to cancer progression. Mitophagy, the selective elimination of mitochondria by autophagy, monitors and maintains mitochondrial health and integrity, eliminating ROS-producing mitochondria. However, mechanisms underlying mitophagic control of mitochondrial homeostasis under basal conditions remain poorly understood. Gp78 E3 ubiquitin ligase is an endoplasmic reticulum membrane protein that induces mitochondrial fission and mitophagy of depolarized mitochondria. Here, we report that CRISPR/Cas9 knockout of Gp78 in HT-1080 fibrosarcoma cells increased mitochondrial volume and rendered cells resistant to carbonyl cyanide m-chlorophenyl hydrazone (CCCP)-induced mitophagy. These effects were phenocopied by knockdown of the essential autophagy protein ATG5 in wild-type HT-1080 cells, suggesting that endogenous Gp78 promotes elimination of mitochondria by autophagy in wild-type HT-1080 cells. Application of a spot detection algorithm (SPECHT) to GFP-mRFP tandem fluorescent-tagged LC3 (tfLC3) transfected cells showed increased accumulation of GFP-mRFP-positive autophagic vacuoles upon treatment with Bafilomycin A under basal conditions and in response to CCCP. Gp78 knockout and inhibition of basal autophagic flux by ATG5 knockdown resulted in reduced mitochondrial potential and increased mitochondrial ROS. Live cell analysis of tfLC3 in HT-1080 cells showed the preferential association of autophagosomes with mitochondria of reduced potential. Gp78dependent basal mitophagic flux therefore promotes mitochondrial health and reduces ROS production in cancer cells.
\end{abstract}




\section{INTRODUCTION}

Mitochondrial autophagy (mitophagy) is a process by which mitochondria are selectively targeted for autophagy and lysosomal degradation ${ }^{1-3}$. Mitophagy plays a critical role in cellular health by controlling mitochondrial mass and eliminating damaged or dysfunctional mitochondria ${ }^{4}$. Reactive oxygen species (ROS) are generated in mitochondria or by membrane-bound NADPH oxidase (NOX) and are elevated in tumor cells ${ }^{5,6}$. At high levels, ROS can induce DNA mutations leading to cellular transformation and tumorigenesis. Autophagy and, more specifically, mitophagy play key roles in controlling ROS production and thereby cancer progression ${ }^{7,8}$. Indeed, in response to hypoxia, BNIP3/NIX/FUNDC1-induced mitophagy has been shown to reduce cellular ROS levels and limit metastasis 2, 9, 10. However, the relationship between mitophagy and ROS production and its regulation in cancer remains complex and not fully elucidated ${ }^{11}$.

Several ubiquitin-dependent molecular pathways have been described to mediate mitophagy, including the well-characterized PINK1/Parkin pathway, closely associated with Parkinson's disease ${ }^{12}$. In response to mitochondrial damage, including ROS, loss of mitochondrial inner membrane potential prevents removal of PTEN-induced putative kinase 1 (PINK1) from the outer mitochondrial membrane (OMM); PINK1, through phosphorylation, recruits and activates the Parkin E3 ubiquitin ligase to damaged mitochondria, triggering recruitment of the autophagy machinery, including the autophagosome-associated protein, microtubule-associated protein 1A/1B-light chain 3 (LC3/ATG8), and autophagosome formation 13-16. Lipidation transforms cytosolic LC3-I to membrane associated LC3-II promoting formation of the phagophore that engulfs dysfunctional mitochondria into autophagosomes for delivery to lysosomes for degradation ${ }^{17}$. However, many cell lines do not express Parkin, tissue expression of Parkin is varied, and mitophagy independent of Parkin and/or PINK1 has been reported ${ }^{18-20}$. Despite the focus on the role of PINK1 and Parkin in damage-induced mitophagy ${ }^{21-24}$, both are dispensable for basal mitophagy in various in vivo systems ${ }^{19,25}$. Other molecular mechanisms must therefore regulate basal mitophagy.

Other ubiquitin ligases such as Mul1, MARCH, SMURF, HUWE1, RNF185 and Gp78 have been reported to function independently or in parallel with PINK1/Parkin 26-32. PINK1-dependent, Parkin-independent mitophagy pathways include the synphilin/SIAH1 and the Mulan pathways ${ }^{28,}$ 29, 33. However, synphilin is primarily expressed in the brain and whether Mulan functions downstream of or independently of PINK1 remains to be determined ${ }^{1}$. Gp78 is a key E3 ubiquitin ligase in the endoplasm reticulum (ER) ${ }^{34,35}$ that has been reported to exhibit both pro-metastatic and tumor suppressor properties 36,37 . Upon mitochondrial depolarization, Gp78 degrades mitofusin $30,38,39$. Gp78 degradation of outer mitochondrial membrane proteins form mitoplasts that interact with the endoplasmic reticulum and are degraded by reticulophagy ${ }^{40}$. Gp78 function in mitophagy has been based primarily on loss of mitochondrial mass upon Gp78 overexpression in the presence of the mitochondrial oxidative phosphorylation uncoupler CCCP ${ }^{30,38-40}$. Defining 
the role of Gp78, and other mitophagy effectors, in tumor cell mitophagy requires study of Gp78 knockout cells and of basal, and not damage-induced, mitophagy.

As a dynamic, multistep process, autophagic activity is best measured by transit through the path, or autophagic flux ${ }^{41}$; however, measuring basal autophagy, or autophagic flux in the absence of autophagy inducers, is particularly challenging. Mitophagy is monitored through loss of mitochondrial mass; reporters like Mito-Keima and Mito-Qc report on delivery of mitochondrial proteins to lysosomes and have proven invaluable to monitor mitophagic flux to lysosomes in cells and tissues ${ }^{19,42-44}$. However, these approaches report on late stages, i.e. lysosomal delivery, of mitophagy. Approaches to measure earlier stages of autophagic flux include analysis of phagophore- and autophagosome-associated LC3B-II and targeted knockdown or knockout of essential autophagy proteins ${ }^{45}$. Western blotting of expression levels of autophagy proteins, such as LC3B-II, report on autophagic activity in a cell population but are limited in their ability to directly measure flux ${ }^{46}$. Fluorescent expression reporters such as GFP-LC3 and GFP-mRFP tandem fluorescent-tagged LC3 (tfLC3) have been used to study autophagic flux in cells and tissues 19, 42-44. However, analysis and interpretation of the acquired data remain challenging. Segmenting LC3 puncta from cytoplasmic LC3 fluorescence is difficult, such that, in some cases, manual counting of LC3 puncta is required ${ }^{45}$.

Here, using CRISPR/Cas knockout of Gp78 in HT-1080 fibrosarcoma cells together with the application of the spot detection algorithm SPECHT ${ }^{47}$ to monitor flux of the autophagosome reporter tfLC3, we show that endogenous levels of Gp78 regulate not only damage-induced mitophagy but also the homeostasis of healthy mitochondria in basal conditions. Basal Gp78dependent mitophagic flux is selectively associated with reduced potential mitochondria and promotes maintenance of a healthy mitochondrial population and limits ROS production.

\section{RESULTS}

\section{Deficient mitophagy in Gp78 knockout HT-1080 cells}

The HT-1080 fibrosarcoma cell line expresses high levels of Gp78 protein and has been extensively used for the study of Gp78 $37,48,49$. We previously used stable miRNA and inducible lenti-shRNA approaches to post-transcriptionally knockdown Gp78 mRNA 30, 49-51. We now applied the CRISPR/Cas9 technique to knockout Gp78 using two different guided RNA sequences: gRNA1 that targets and eliminates the ATG start codon; and gRNA2 that induces a frameshift sixteen amino acids downstream of the ATG start codon (Figure 1 A). Complementary gRNA1 or gRNA2 oligos were annealed and cloned in the GeneArt-OFP plasmid, sequence verified and transfected into HT-1080 cells. We screened sixty clones by Western blotting and obtained 38 Gp78 knockout clones. Three clones showing complete absence of Gp78 from each 
gRNA were chosen (gRNA1: clones \#3, 4 and 7; gRNA2: clones \#13, 36 and 41) for further experimentation. From isolated genomic DNA for each clone, the DNA fragment flanking Exon1 of Gp78 was PCR amplified, cloned and sequenced. For gRNA1 clones \#3 and \#4 the G was deleted from the ATG start codon while for clone \#7 an additional T was inserted in the start codon generating ATTG. For all three gRNA2 clones $(\# 13,36,41)$, a T was inserted at amino acid 16 (CCTA to CCTTA) causing a frameshift mutation. Probing Western blots with anti-Gp78 polyclonal antibody showed the complete absence of Gp78 in all six gRNA1 and gRNA2 clones compared to wild-type HT-1080 cells (Figure 1 A).

Gp78 CRISPR/Cas9 knockout HT-1080 cells labeled for the inner mitochondrial protein ATPB synthase all showed extended mitochondrial networks compared to wild-type HT-1080 cells. Quantification of total mitochondrial volume from 3D spinning disk confocal stacks showed a significant 2 -fold increase in mitochondrial volume in the Gp78 knockout clones relative to wildtype HT-1080 cells (Figure 1 B). Rescue of Gp78 KO HT-1080 cells by transfection of wild-type Gp78, but not Gp78 containing a point mutation in the RING finger domain required for Gp78 ubiquitin ligase activity ${ }^{34,52}$, reduced mitochondrial volume confirming that Gp78 ubiquitin ligase activity controls mitochondrial volume in HT-1080 cells (Supp. Figure 1). To determine if Gp78 KO specifically affected mitophagy, we assessed the impact of the mitochondrial membrane potential decoupler, CCCP $(10 \mu \mathrm{M} ; 24 \mathrm{~h})$ on mitochondrial volume in wild-type and Gp78 knockout HT-1080 cells. CCCP treatment induced mitochondrial fragmentation and a significant reduction in mitochondrial volume in wild-type HT-1080 cells. However, while CCCP treatment induced fragmentation of mitochondria of Gp78 knockout HT-1080 cells, the elevated mitochondrial volume of the Gp78 knockout clones was not reduced (Figure $1 \mathrm{~B}$ ). This suggests that endogenous Gp78 is required for damage-induced mitophagy in HT-1080 cells.

To specifically determine whether autophagy was responsible for the reduced mitochondria levels of Gp78 knockout cells, we knocked down the essential autophagy gene ATG5 in wild-type HT1080 cells and representative Gp78 knockout g1-4 and g2-41 clones, obtained with gRNA1 or gRNA2, respectively. By Western blot analysis, mitochondrial ATPB synthase levels showed a significant two-fold elevation in both Gp78 knockout clones relative to wild-type HT-1080 cells (Figure $2 \mathrm{~A}$ ), consistent with the increased mitochondrial volume of these cells (Figure $1 \mathrm{~B}$ ). Upon ATG5 siRNA knockdown, by Western blot ATPB synthase levels in HT-1080 cells increased to those of Gp78 knockout cells (Figure 2A). Similarly, ATG5 KD significantly increased mitochondrial volume by 3D spinning disk confocal analysis of both untreated and CCCP-treated wild-type HT-1080 cells (Figure 2B). ATG5 KD did not affect mitochondrial volume in the Gp78 knockout clones in the absence or presence of CCCP. This suggests that the increased mitochondrial volume of Gp78 knockout cells, in the absence of CCCP, is due to inhibition of active Gp78-dependent mitophagy in wild-type HT-1080 cells. 


\section{Gp78 enhances CCCP-induced mitophagic flux}

We then undertook to determine whether Gp78 regulates autophagic flux and more specifically mitophagic flux. We monitored accumulation of the membrane associated LC3B-II by Western blot upon inhibition of lysosomal acidification and degradation using BafA1. Cells were treated for 4 hours with CCCP to induce mitophagy or serum-starved to induce macroautophagy. With increasing time of BafA1 incubation, cells treated with DMSO or serum-starved showed increased LC3B-II accumulation in both HT-1080 cells and the g1-4 and g2-41 Gp78 knockout clones. Quantification of flux, based on the slope of LC3B-II band density relative to $\beta$-actin over time, showed no significant differences between the three cell lines at basal levels. Upon treatment with CCCP (mitophagy inducer), LC3B-II flux was clearly observed in HT-1080 cells but not in the Gp78 knockout clones. These data demonstrate that Gp78 is selectively required for damageinduced mitophagic flux, consistent with the inability of CCCP to induce mitochondrial loss in Gp78 knockout cells (Figure 1). However, this Western blot analysis did not detect differences in basal mitophagy that could explain the increased mitochondrial mass of Gp78 knockout cells, perhaps related to previously reported limitations of Western blot analysis of autophagic proteins for detection of autophagic flux ${ }^{46,53}$.

\section{Gp 78 promotes basal mitophagic flux}

To develop a more specific and sensitive assay to measure basal autophagic flux, we used the tandem fluorescent-tagged tfLC3 in which LC3 is linked to both GFP and mRFP1; the more pHsensitive GFP is a marker for early autophagosomes while the less $\mathrm{pH}$-sensitive mRFP remains fluorescent in acidic autophagolysosomes ${ }^{54}$. Stably expressed tfLC3 in HT-1080 cells presented a diffuse cytosolic distribution in both the GFP and mRFP channels with a few puncta corresponding to autophagic vacuoles (Figure $4 \mathrm{~A}$ ). Increased accumulation of mRFP puncta relative to GFP puncta, due to mRFP's $\mathrm{pH}$-insensitive fluorescence and resistance to lysosomal degradation, is indicative of ongoing basal autophagy in these cells ${ }^{55,56}$. Dually labeled GFPmRFP puncta correspond to neutral $\mathrm{pH}$ autophagosomes; accumulation of dually labeled GFPmRFP puncta upon short-term BafA1 treatment reflects the accumulation of intact tfLC 3 in acidic autophagosomal compartments and is therefore a measure of autophagic flux. Upon 4-hour CCCP treatment in the presence of BafA1 for the final two hours, conditions that induce a robust mitophagic flux response (Figure 3), an increase in both GFP and mRFP labeled puncta was observed, including multiple overlapping puncta (Figure $4 \mathrm{~A}$ ).

However, quantifying LC3 puncta amongst the diffuse fluorescence signal of cytoplasmic LC3 is challenging ${ }^{45}$. Application of the SPECHT spot detection algorithm was used to robustly identify LC3 puncta ${ }^{47}$. To remove puncta smaller than the $250 \mathrm{~nm}$ resolution of diffraction limited confocal microscopy that could not correspond to 300-1000 nm autophagosome precursors or phagophores 57 , we applied a size filter that removed any puncta of 25 pixel area (pixel size $=56.6 \mathrm{~nm}$ ) or less. 
Overlapping GFP and mRFP puncta (at least one pixel) were considered to be dually-labeled GFPmRFP puncta (spots) and are highlighted in yellow and also shown overlaid with the mask of the mitochondrial signal (Figure $5 \mathrm{~A}$ ).

To identify the intensity-independent $\mathrm{z}$ score threshold (see Materials and Methods - spot detection) value that most accurately detects LC3-positive autophagosomes, we performed a parameter sensitivity study on $z$. We tested the impact of $z$ on differential association of autophagosomes with mitochondria between DMSO and CCCP+BafA1 treated cells, known to present a highly significant difference in autophagic flux (Figure 3). A total of $21 \mathrm{z}$-values were tested, with values of $z>2$ resulting in empty image masks. Given that we have 4 groups $(\mathrm{CCCP} \pm \mathrm{BafA}, \mathrm{DMSO} \pm \mathrm{BafA}$ ), we have 6 possible combinations (from 4 choose 2 ) and 2 cell lines $(\mathrm{m}=6 \times 2 \times 21=252$ hypothesis tests). To account for multiple testing correction, we applied the Bonferroni correction with a corrected $\alpha$ of $\alpha / 252$. Bonferroni allows computation of the correction without testing all hypotheses explicitly; it is however strongly conservative risking false negatives which, in a parameter sensitivity study, is not an unwanted trait. We applied the Kruskal nonparametric test to compare if the difference between the 2 conditions is significant and more importantly, consistent across the parameter range. Low z-values causes higher recall of objects, which possibly skews the results towards significance. At the other end, high z-values erode the size and diminishes the counts of detected puncta (Figure 4 B, Supp. Figure 2). Application of SPECHT to size filtered LC3 at $\mathrm{z}=1.75$ dramatically improved LC3 puncta detection, significantly detecting differential expression of dually labeled GFP-mRFP tfLC3 puncta between control and $\mathrm{CCCP} / \mathrm{BafA} 1$ treated cells and accurately reproducing puncta distribution (Figure $4 \mathrm{C}$, Supp. Figure 2). We used $z=1.75$. that shows a highly significant difference between DMSO and $\mathrm{CCCP}+\mathrm{BafA} 1$ conditions (Figure $4 \mathrm{C}$ ). Two hours BafA1 treatment induced dually labeled GFPmRFP tfLC3 puncta not only in CCCP-treated cells but also in untreated cells, indicative of active basal autophagy in HT-1080 cells. BafA1-induced GFP-mRFP tfLC3 puncta encompass both neutral autophagosomes and acidic autophagolysosomes, that following the sequestration event will move away from mitochondria. About 50\% of BafA1-induced GFP-mRFP tfLC3 puncta were located within the resolution limit of 5 pixels $(\sim 250 \mathrm{~nm})$ to mitochondria for both untreated and CCCP treated cells (Figure 4D), indicative of a similar mitochondria association of autophagic vacuoles under basal and CCCP conditions. SPECHT analysis of tfLC3 therefore detects both basal and damage-induced mitophagic flux in HT-1080 cells.

Having established conditions to detect mitochondrial-associated autophagosomes in HT-1080 cells in response to CCCP, known to induce mitophagy in HT-1080 cells, we then applied this approach to HT-1080 and Gp78 knockout g2-41 cell lines in the absence of CCCP. Time course analysis of tfLC3 expression showed a significant increase in dual labeled GFP-mRFP LC3 spots positive for GFP and mRFP upon 4 hours BafA1 treatment of HT-1080 cells that showed a high degree of proximity to mitochondria (Figure 5 A). Dual labeled GFP-mRFP LC3 spots showed no increase in the absence of BafA1 or in $\mathrm{g} 2-41 \mathrm{Gp} 78 \mathrm{KO}$ cells in the presence of BafA1. The BafA1- 
induced increase in GFP-mRFP-positive tfLC3 spots in the absence of CCCP is indicative of basal autophagic flux and association of tfLC3 puncta to mitochondria suggestive of basal mitophagy. Inhibition of autophagy by siRNA knockdown of the essential autophagy gene ATG5 (siATG5) prevented BafA1 induction of GFP-mRFP-positive tfLC3 puncta, confirming that this assay is accurately reporting on basal autophagic processes (Figure 5 B). Basal mitophagy of HT-1080 cells is therefore Gp78-dependent.

\section{Gp78 promotes mitochondrial health and limits ROS production}

We then tested whether Gp78-dependent basal mitophagy impacts mitochondrial potential, and mitochondrial ROS. HT-1080 and g2-41 Gp78 KO cells were incubated with MitoView 633, a potential-dependent mitochondrial reporter, or MitoSox Red, a mitochondrial ROS reporter and imaged by live cell microscopy. MitoView 633 labeling of HT-1080 cells was significantly reduced by both Gp78 KO and ATG5 siRNA knockdown (Figure $6 \mathrm{~A}$ ). In contrast, Gp78 KO and ATG5 siRNA significantly increased MitoSox Red labeling of mitochondrial ROS (Figure 6 B). The parallel effect of both Gp78 KO and ATG5 knockdown indicates that basal mitophagy promotes mitochondrial health and limits ROS production in HT-1080 cells. That siATG5 did not affect either MitoView or MitoSox labeling in Gp78 KO HT-1080 cells suggests that Gp78 is the key regulator of mitochondrial quality control in these cancer cells.

Live cell time lapse imaging of tfLC3-expressing HT-1080 cells labeled for MitoView 633 was performed to determine if autophagosomes specifically associate with damaged mitochondria, i.e. those showing reduced mitochondrial potential. Cells presenting multiple GFP LC3 puncta were imaged by spinning disk confocal and image stacks, 7 images at $500 \mathrm{~nm}$ spacing, to cover the depth of the cell and minimize overlap of autophagosomes in different Z-sections, were acquired every minute over 40 minutes. SPECHT analysis identified dual-labeled GFP-mRFP tfLC3 puncta, corresponding to phagophores or autophagosomes, in every frame. Representative time lapse images are presented in Figure 7 A. Dual-labeled GFP-mRFP tfLC3 puncta were associated with MitoView 633 labeled mitochondria. To determine if autophagosomes were more closely associated with lower potential mitochondria, we quantified the average intensity of MitoView 633 positive pixels overlapping GFP-RFP-positive tfLC3 puncta relative to average MitoView 633 intensity in the average of all MitoView positive pixels in the complete mitochondrial segment overlapping the autophagosome or in the whole cell. As can be seen in Figure 7 A, MitoViewpositive pixels overlapping GFP-mRFP-positive tfLC3 puncta presented a below average mitochondrial potential. Time lapse series acquired every 10 seconds show the dynamic association of GFP-mRFP-positive tfLC3 puncta with low potential mitochondria (Figure 7B; Supplemental Video 1). These data show that autophagosomes in HT-1080 cells associate with damaged mitochondria, thereby contributing to maintenance of mitochondrial health of these cells. 


\section{DISCUSSION}

Here, using CRISPR/Cas9 knockout in the HT-1080 fibrosarcoma cell line, we show that the Gp78 ubiquitin ligase controls basal mitophagic flux and thereby mitochondrial health and ROS production. Increased mitochondrial volume of HT-1080 cells upon Gp78 knockout and increased mitochondrial mass upon ATG5 siRNA knockdown was indicative of increased basal mitophagy. Basal autophagy maintains cellular homeostasis through the sequestration and subsequent removal of bulk cytoplasmic constituents by delivery to lysosomes for degradation. Functional consequences of defective basal autophagy in ATG5 knockout mice include thyrocyte death, neurodegeneration and increased oxidative stress associated with elevated ROS in these cell populations, possibly because of the accumulation of damaged mitochondria ${ }^{58,59}$. Basal autophagy has been shown to selectively target subcellular components such as lipids and organelles ${ }^{60-63}$. The deficient mitochondrial metabolic activity and increased ROS production associated with the impaired basal mitophagy of Gp78 knockout HT-1080 cells provides a definitive link between basal mitophagy and maintenance of mitochondrial health.

The basis for Gp78 selective targeting of reduced potential mitochondria for mitophagy remains unclear. Gp78 overexpression recruits LC3 to mitochondria-associated ER ${ }^{30}$ and Gp78 has been shown to selectively regulate rough ER-mitochondria contacts ${ }^{49}$. Also, Gp78 has been recently shown to be involved in ERphagy, and selective degradation of the outer mitochondrial membrane, forming mitoplasts and triggering mitophagy ${ }^{64}$. The relationship between Gp78-dependent mitoplast formation, the ER and the dynamic movement and maturation of LC3-positive autophagosomes in proximity to mitochondria that we report here remains to be determined. Considering the dispensable role of Parkin and PINK1 in basal mitophagy in vivo ${ }^{19,25}$, further analysis of the role of Gp78-dependent basal mitophagy in tissue development and cancer progression is warranted.

Tandem tfLC3 reports on autophagic flux at the level of autophagosome maturation due to the differential sensitivity of GFP and mRFP to acid quenching of fluorescence and degradation in lysosomes. mRFP fluorescence is therefore a reporter of LC3 accumulation in autophagolysosomes while dual GFP-mRFP fluorescence is a reporter of LC3B-II association with phagophores and neutral early autophagosomes. Increased dual GFP-mRFP tfLC3 fluorescence upon BafA1 neutralization of acidic autophagolysosomes, enabling GFP fluorescence and preventing GFP degradation, is therefore an indicator of autophagic flux. However, the presence of cytosolic tandem LC3 fluorescence makes detection of GFP-positive autophagosomes difficult by standard segmentation approaches ${ }^{45}$. In accordance, a prior study of basal autophagy in HEK293 cells relied on manual counting of LC3 puncta ${ }^{65}$. By applying the spot detection stage of the SPECHT algorithm ${ }^{47}$, we report the sensitive and robust detection of LC3 puncta. Using CCCP induction of mitophagic flux in HT-1080 cells to establish detection parameters, we used BafA1 inhibition of autophagosome maturation to demonstrate the presence of basal autophagic 
flux in HT-1080 cells but not in Gp78 knockout HT-1080 cells. Mitochondrial association of BafA1-induced autophagosomes paired with the reduced mitochondria volume and potential and the increased mitochondrial ROS upon inhibition of autophagy using siATG5 demonstrates that HT-1080 cells undergo active basal mitophagy. In contrast to the Mito-Keima and Mito-QC reporters report directly on mitophagic flux, detecting delivery of mitochondrial proteins to lysosomes 19,43,44, 66, live cell time lapse analysis of tfLC3 in HT-1080 cells allowed us to demonstrate that autophagosomes associate with reduced potential mitochondria, confirming a role for mitophagy in mitochondrial quality control. The enhanced sensitivity of detection of tandem tfLC3 using SPECHT, even when channels differ markedly in signal to noise ratio, renders this a valuable tool to study basal autophagy in various cell and tissue systems.

ROS generation shows a complex association with tumor progression. ROS are critical for tumor initiation and progression acting through activation of oncogenic signaling and DNA mutation; at the same time, increasing ROS production eliminates cancer cells by inducing cell death programs 67. Regulation of mitochondrial ROS production by BNIP3, a hypoxia-induced mitophagy regulator, suppresses breast cancer progression ${ }^{68,69}$. The formation of spontaneous liver tumors after 12 months in Gp78 knockout mice supports a tumor suppressor function for Gp78 ${ }^{36}$ that is consistent with Gp78-dependent mitophagic control of ROS production. However, Gp78 knockdown in the HT-1080 cells studied here did not affect tumor growth but did inhibit metastasis 37; further, impaired metastasis was rescued by wild-type Gp78 but not a RING finger Gp78 mutant with deficient ubiquitin ligase activity that does not induce mitophagy ${ }^{30,37}$. This suggests that Gp78 metastasis promotion is not necessarily related to mitophagy and ROS production. Indeed, Gp78 was shown to promote metastasis though targeted degradation of the metastasis suppressor KAI1 37 and Gp78 downregulation of KAI1 shown to be associated with mammary gland hyperplasia ${ }^{70}$. This suggests multiple roles for Gp78 in cancer progression, including but not limited to regulation of ROS production through mitophagy.

\section{MATERIALS AND METHODS}

\section{Antibodies and reagents}

Anti-ATPB pAb/mAb (ab128743/ab5432) was purchased from Abcam (Cambridge, MA, USA), anti-ATG5 pAb (NB110-53818) from Novus Biologicals (USA), anti-Gp78 pAb (16675-1-AP) from Proteintech (USA) and anti-LC3B pAb (2775S) from Cell Signaling. Anti- $\beta$-Actin mAb (A5441), tissue culture grade DMSO (D2650), Bafilomycin A1 (Cat\# C1988) and CCCP (C2759) were purchased from Sigma-Aldrich (USA). mRFP-GFP tandem fluorescent-tagged LC3 (tfLC3) was a gift from Tamotsu Yoshimori (Addgene plasmid\# 21074; http://n2t.net/addgene:21074; RRID:Addgene_21074) ${ }^{54}$. MitoTracker Deep Red FM, Live Cell Imaging Solution (A14291DJ), Glucose Solution (A2494001), MitoSOX Red (M36008) and MEM Non-Essential Amino Acids 
Solution (11140050) were purchased from ThermoFisher (USA). MitoView 633 (\#70055) was purchased from Biotium (USA).

\section{Cell lines and CRISPR/Cas9 knockout of Gp78}

The HT-1080 fibrosarcoma cell line was acquired from ATCC, authenticated by Short Tandem Repeat (STR) profiling at the TCAG Genetic Analysis Facility (Hospital for Sick Kids, Toronto, ON, Canada www.tcag.ca/facilities/geneticAnalysis.html), tested regularly for mycoplasma infection by PCR (ABM, Richmond, BC, Canada) and maintained in RPMI 1604 media supplemented with $10 \%$ FBS and $1 \%$ L-Glutamine in a $37^{\circ} \mathrm{C}$ incubator with $5 \% \mathrm{CO}_{2}$.

GeneArt-CRISPR/Cas9 Nuclease vector with OFP (Orange Fluorescence Protein) kit (A21174) was from Life Technologies (Invitrogen, USA). We used http://crispr.mit.edu to design guided RNAs and http://www.rgenome.net/cas-offinder/ (RGEN tools) to check them for off-target effects and used the following oligonucleotides for guide RNA1 (5'-CAC CGG AGG AAG AGC AGC GGC ATG G-3', 5'-AAA CCC ATG CCG CTG CTC TTC CTC C-3') and guide RNA2 (5'CAC CGG CCC AGC CTC CGC ACC TAC A-3', 5'-AAA CTG TAG GTG CGG AGG CTG GGC C-3'). The guide RNAs were in vitro annealed, cloned into the GeneArt linear vector according to the supplier's protocol and sequence verified prior to transfection into HT-1080 cells. Sequence verified gRNA1 or gRNA2 containing GeneArt-CRISPR/Cas9 Nuclease vector with OFP were transiently transfected into HT-1080 cells, plated 24 hours previously, using Effectene transfection reagent (301425, Qiagen, USA). After 36 hours incubation, cells were harvested and genomic DNA isolated to perform GeneArt Genomic Cleavage Detection assay (A24372, Invitrogen, USA) to check cleavage efficiency. Once cleavage efficiency was confirmed, HT-1080 cells were replated for 36 hours, trypsinized, FACS sorted and OFP expressing cells were singly plated in 96 well pates by serial dilution. Single colonies were replicated in 12 well plates; one set was frozen and stored in liqN2 and the other set subjected to lysate preparation, SDS-PAGE and Gp78 western blot analysis. Arbitrarily chosen representative clones (g1-3, g1-4, g1-7; g2-13, g236, g2-41) from both gRNAs were expanded, tested for mycoplasma and stored as multiple freezedowns. From isolated genomic DNA, an approximate 800bp fragment flanking Exon1 of Gp78 was PCR amplified using Q5 (Qiagen, USA) the following primer set (Forward: 5'-CTG GAG GCT ACT AGC AAA-3', Reverse: 5'-ATG TGG CCC AGT ACC T-3') and TA cloned. At least ten clones were sequenced from each to confirm INDEL.

HT-1080 and Gp78 CRISPR/Cas9 knockout clones were grown only up to six passages. Cells were passed every 48 hours at a density of 300,000 cells per $10 \mathrm{~cm}$ petri dish, rinsed every 24 hours with $10 \mathrm{ml}$ of PBS and supplied with $10 \mathrm{ml}$ of fresh complete medium. HT-1080 cells and the g2-41 Gp78 CRISPR/Cas9 knockout clones were stably transfected with mRFP-GFP tandem fluorescent-tagged LC3 (tfLC3) plasmid using Effectene (Cat. \#301425, Qiagen, USA) following the manufacturer's protocol. After 24 hours incubation, transfected cells were selected against 
G418 (400ug/ml) for about 14 days. The resistant cell population was pooled and maintained in $50 \mu \mathrm{g} / \mathrm{ml} \mathrm{G} 418$.

\section{siRNA knockdown, plasmid transfection and western blotting}

siControl and siATG5 (Cat\# D-001810-01-05, Cat\# L-004374-00-0005) were purchased from Dharmacon and transiently transfected wherever indicated to wild-type HT-1080 cells or g1-4 or g2-41 Gp78 CRISPR/Cas9 knockout clones using Lipofectamine 2000 (Cat\# 11668019, Invitrogen, USA) following the manufacturer's protocol. All siRNA transfection experiments were for 48 hours and treatments were performed 24 hours post siRNA transfection. Alternatively, cells were transiently transfected with mammalian protein expressing plasmids using Effectene (Qiagen, Germany) following the manufacturer's protocol. Where indicated, cells were treated with $10 \mu \mathrm{M}$ of CCCP or a corresponding volume of DMSO as control 24 hours prior to fixation or harvesting cells. Western blotting was performed as previously described using Horseradish Peroxidase (HRP)-conjugated secondary antibody followed by addition of ECL (GE Healthcare Bio-Sciences Corp., USA) to reveal chemiluminescence ${ }^{70}$. Densitometry quantification was done using ImageJ (https://imagej.nih.gov/ij/docs/faqs.html\#cite) software.

\section{Fluorescent labeling of mitochondria}

For immunofluorescent labeling, cells were: 1) fixed with 3.0\% PFA for 15 minutes at room temperature and washed with PBS-CM (phosphate buffer solution supplemented with $1 \mathrm{mM}$ $\mathrm{CaCl} 2$ and $10 \mathrm{mM} \mathrm{MgCl} 2$ ); 2) permeabilized with $0.2 \%$ Triton X-100 for 5 minutes and washed with PBS-CM; 3) blocked with 1\% BSA for 1 hour at room temperature; 4) labeled with antiATPB for one hour followed by washing with PBS-CM; 5) incubated with secondary antibodies for 1 hour followed by washing with PBS-CM; and 6) mounted in ProLong Diamond (ThermoFisher) and cured for 24 hours at room temperature before imaging. Confocal image stacks were obtained on a III-Zeiss spinning disk confocal microscope with either Zeiss PlanApochromat 63X/1.2NA or 100X/1.4NA oil objectives using SlideBook 6.0 image acquisition and analysis software (Intelligent Imaging Innovation Inc). ATPB label was thresholded from 3D images to measure mitochondrial volume with SlideBook 6.0 image analysis software.

To assess the impact of Gp78-dependent basal mitophagy on mitochondrial health and mitochondrial ROS, wildtype HT-1080 and the g2-41 Gp78 CRISPR/Cas9 knockout clone were plated into an Ibidi chamber for 24 hours. Cells were then transiently transfected with siRNA targeting ATG5 for 48 hours and labelled with either mitochondrial health sensor dye MitoView633 or mitochondrial ROS dye MitoSOX, at concentrations of $50 \mathrm{nM}$ and $2.5 \mu \mathrm{M}$, respectively, for half an hour, washed $3 \mathrm{X}$ with warm PBS and incubated in Molecular Probes Live Cell Imaging Solution. Live-cell imaging was performed at $37^{\circ} \mathrm{C}$ with a Leica TCS SP8 confocal microscope with a 100×/1.40 Oil HC PL APO CS2 objective (Leica, Wetzlar, Germany) equipped 
with a white light laser, HyD detectors, environmental chamber and Leica Application Suite X (LAS X) software. Images were analyzed using ImageJ software to identify integrated densities of mitochondrial objects as well as the total area of the mitochondrial label, per cell.

\section{Mitophagic flux assays}

In order to study mitophagic flux in HT-1080 or Gp78 CRISPR/Cas9 knockout (g1-4, g2-41) cells, early passage cells $(420,000$ cells per well) were plated in six well plates for 20 hours, then washed with 1X PBS and treated with DMSO or CCCP in regular medium or medium lacking serum for 4 hours. For each treatment, cells were challenged with $100 \mathrm{nM}$ of BafA1 (Sigma) for 0, 30, 60 or 120 minutes prior to the end of the 4-hour incubation period. Incubation was stopped by washing cells with ice-cold 1X PBS; cells were then harvested on ice lysed with M2-Lysis buffer 71 supplemented with phosphatase and protease inhibitors tablets (Roche), and lysates ran on 15\% SDS-PAGE at constant voltage (75V for fifteen minutes followed by $90 \mathrm{~V}$ for 2 hours). Separated proteins were electrotransferred onto $0.2 \mu$ pore size PVDF membrane (BioRad), fixed with $0.1 \%$ glutaraldehyde in PBST (0.2\%) for 30 minutes, blocked with 5\% milk in PBST and immunoprobed for LC3B-I and II and $\beta$-Actin. LC3B-II and $\beta$-actin bands were densitometrical quantified using ImageJ software, normalized and statistically analyzed.

To monitor autophagic flux with tfLC3, stably transfected HT-1080 and Gp78 CRISPR/Cas9 knockout g2-41 cells were plated overnight and then treated with either DMSO or CCCP for 4 hours and with or without $100 \mathrm{nM}$ BafA1 for the final 2 hours of the incubation period. Mitochondria were labelled with MitoTracker Deep Red FM half an hour prior to the end of the total incubation period. After incubation, cells were gently washed $3 \mathrm{X}$ with warm PBS and then incubated in warm Live Cell Imaging Solution just prior to image acquisition. Live-cell imaging was performed using Leica TCS SP8 confocal microscope with a 100×/1.40 Oil HC PL APO CS2 objective (Leica, Wetzlar, Germany) equipped with a white light laser, HyD detectors, and Leica Application Suite X (LAS X) software. Image acquisition was performed in a temperaturecontrolled system set to $37^{\circ} \mathrm{C}$.

For time lapse imaging, HT-1080 cells expressing tfLC3 were plated in ibidi chambers in Molecular Probes Live Cell Imaging Solution supplemented with 10\% FBS, L-glutamine, Dglucose, and MEM Non-Essential Amino Acids Solution and labeled with MitoView prior to imaging at $37^{\circ} \mathrm{C}$ with the $100 \mathrm{X} / \mathrm{NA} 1.45 \mathrm{PL}$ APO objective (Zeiss) of a 3i Yokogawa X1 spinning disk confocal. Image stacks of 7 images with a $500 \mathrm{~nm} \mathrm{Z} \mathrm{spacing} \mathrm{were} \mathrm{acquired} \mathrm{every} \mathrm{minute} \mathrm{for}$ 40 minutes with a QuantEM 512SC Photometrics camera. Average intensity of MitoView positive pixels overlapping each GFP-mRFP-positive tfLC3 puncta was assessed relative to average intensity of all MitoView-positive pixels in either the adjacent segmented mitochondria or in the cell. 


\section{tfLC3 spot detection analysis (SPECHT)}

To identify tfLC3 labeled autophagic vacuoles (autophagosomes), we applied the SPECHT object detection method, that is consistent across channels and robust to intensity variations ${ }^{47}$. SPECHT evolved from the ERGO software for density detection in single molecule localization microscopy 72 and accepts as input a confocal image and produces, for each channel, a binary mask of detected fluorescent marker concentrations (spots or puncta). SPECHT leverages the Laplacian-ofGaussian (LoG) object detection method, but ensures detection is adaptive to the image intensity distribution by computing an automatic threshold to postprocess LoG detected objects. The user can express a preference for recall or precision, which SPECHT then translates into channel/image specific threshold values. This preference is referred to in this manuscript as ' $\mathrm{z}$-value'. A higher value increases precision, at the cost of recall. A lower value can result in higher recall, at cost of precision. To ensure no artificial objects are introduced, the isotropic Gaussian std. dev. was set to round $(\operatorname{precision} / 2)=3$ pixels (pixel size $=56.6 \mathrm{~nm}$ ). Objects with area smaller than 25 pixels were removed to avoid false counting of artifacts below the precision limit of the acquisition. Distances between objects were measured using Euclidean distance (pixels) between the closest edges of nearest objects. Puncta within 5 pixels (ceil(precision)) of mitochondria (i.e. the resolution limit of $\sim 250 \mathrm{~nm}$ ) cannot be distinguished from overlapping puncta and were thus counted as overlapping. The area of objects is represented by pixel mask counting. When a red mRFP and green GFP object (puncta) shared a non-zero intersection, the union of the red-green overlapping puncta were considered to correspond to early, neutral $\mathrm{pH}$ autophagosomes. Colocalized GFPmRFP tfLC3 puncta upon BafA1 treatment encompass acidic autophagolysosomes and an increase in GFP-mRFP tfLC3 puncta following BafA treatment is a measure of autophagic flux. We also quantified the number of BafA1-induced GFP-mRFP overlapping tfLC3 puncta within 5 pixels of mitochondria.

To ensure single cell analysis, ROIs encompassing complete, individual cells within the field of view were manually segmented (Figure 6). While this was feasible for the single time point analysis, for the time lapse series (Figure 7), cells were moving and we therefore added an automated preprocessing stage to obtain cell segmentation masks. Input to SPECHT was a sequence of 2D images (1 Z-slice), 3 channels per timepoint. We recover the outline by applying a median filter (window sizes 3x3, 5x5, 9x9) after filtering out the $90 \%$ intensity distribution quantile, binarizing the resulting image, and detecting disjointed objects separated by black (filtered) background using the connected components algorithm, such that the sole complete cell will be the largest object. To accommodate the highly fluctuating intensity distribution of live cell imaging over time, we enable SPECHT's autotuning mode configured to recover all possible objects (recall/precision ratio $=3.75$ ). To prevent inclusion of false positives we: 1 ) compute the effect size (Cohen's d) of its intensity distribution with respect to that of the cell and discard any object with a negative effect size; 2) we use the heuristic that the local maxima contained within each detected object, should be a statistical outlier with respect to the overall intensity distribution 
(Q3 + 1.5 IQR, respectively $3^{\text {rd }}$ quartile and interquartile range) and discard objects that have a maximum intensity that is not at the extremum of the intensity distribution; 3) objects of area smaller or equal to 4 pixels are discarded, as they cannot be shown to be observable under the precision of the system ( 2 pixels). To ensure we do not compromise objects at the cell edge we widen the cell mask by a dilation operation 4 times $(2 \mathrm{x}$ system precision of 2 pixels). A closing operation ensures no holes are left in the cell mask should one channel have no or weak labelling in part of the resulting mask. To ensure our segmentation is valid, without the user having to screen each image, we test that the cell mask is consistent across channels. In addition, we disregard processing of any image where the cell mask touches the border of the image. The combination of high recall followed by high precision filtering, ensures a balanced, robust automatic pipeline for object detection designed for the live cell imaging data. For each GFP-mRFP overlapping spot (C12), we compute the mean mitochondria intensity it overlaps relative to the mean intensity of the associated mitochondria segment as well as the mean intensity of all mitochondria segments in each given 2D image (C). We then compute a box plot of ratios 1 and 2 for all C12 objects, for each cell (Figure 7), where each cell is represented by 7 2D images (per Z-slice), over 40 timepoints. Output is saved in csv files for statistical analysis and postprocessing. The processing code is being prepared for open source release (Affero GPLv3), and available upon reasonable request.

\section{Statistical analyses}

One-way ANOVA with Dunnett's multiple comparison test was used for both the fixed and livecell ROS experiments. One-way ANOVA with Tukey's multiple comparison test was used for the live-cell tfLC3 flux, the spinning disk mitochondrial volume experiments and the Western blot flux experiments. A 2 tailed t-test was applied for the siATG5 blots. Statistical analyses were performed using GraphPad Prism 6.0 software.

\section{ACKNOWLEDGEMENTS}

This study was supported by a grant from the Canadian Institutes of Health Research (CIHR Grant PJT-148698) and UBC Fellowships (GG). Imaging was performed in the LSI IMAGING facility of the Life Sciences Institute of the University of British Columbia using infrastructure funded by the Canadian Foundation of Innovation and BC Knowledge Development Fund as well as a Strategic Investment Fund (Faculty of Medicine, University of British Columbia). This research was enabled in part by support provided by Westgrid (https://www.westgrid.ca/) and Compute Canada (www.computecanada.ca). 


\section{REFERENCES}

1. Zimmermann M, Reichert AS. How to get rid of mitochondria: crosstalk and regulation of multiple mitophagy pathways. Biol Chem 2017; 399:29-45.

2. Drake LE, Springer MZ, Poole LP, Kim CJ, Macleod KF. Expanding perspectives on the significance of mitophagy in cancer. Semin Cancer Biol 2017; 47:110-24.

3. Harper JW, Ordureau A, Heo JM. Building and decoding ubiquitin chains for mitophagy. Nat Rev Mol Cell Biol 2018; 19:93-108.

4. Springer MZ, Macleod KF. In Brief: Mitophagy: mechanisms and role in human disease. J Pathol 2016; 240:253-5.

5. Gorrini C, Harris IS, Mak TW. Modulation of oxidative stress as an anticancer strategy. Nat Rev Drug Discov 2013; 12:931-47.

6. Murphy MP. How mitochondria produce reactive oxygen species. Biochem J 2009; 417:113.

7. Mathew R, White E. Autophagy, stress, and cancer metabolism: what doesn't kill you makes you stronger. Cold Spring Harb Symp Quant Biol 2011; 76:389-96.

8. Poillet-Perez L, Despouy G, Delage-Mourroux R, Boyer-Guittaut M. Interplay between ROS and autophagy in cancer cells, from tumor initiation to cancer therapy. Redox Biol 2015; 4:184-92.

9. Zhang H, Bosch-Marce M, Shimoda LA, Tan YS, Baek JH, Wesley JB, et al. Mitochondrial Autophagy Is an HIF-1-dependent Adaptive Metabolic Response to Hypoxia. Journal of Biological Chemistry 2008; 283:10892-903.

10. Zhang J, Ney PA. Role of BNIP3 and NIX in cell death, autophagy, and mitophagy. Cell Death Differ 2009; 16:939-46.

11. Schofield JH, Schafer ZT. Mitochondrial Reactive Oxygen Species and Mitophagy: A Complex and Nuanced Relationship. Antioxid Redox Signal 2021; 34:517-30.

12. Pickrell AM, Youle RJ. The roles of PINK1, parkin, and mitochondrial fidelity in Parkinson's disease. Neuron 2015; 85:257-73.

13. Ordureau A, Heo JM, Duda DM, Paulo JA, Olszewski JL, Yanishevski D, et al. Defining roles of PARKIN and ubiquitin phosphorylation by PINK1 in mitochondrial quality control using a ubiquitin replacement strategy. Proc Natl Acad Sci U S A 2015; 112:6637-42.

14. Lazarou M, Sliter DA, Kane LA, Sarraf SA, Wang C, Burman JL, et al. The ubiquitin kinase PINK1 recruits autophagy receptors to induce mitophagy. Nature 2015; 524:309-14.

15. Sekine S, Youle RJ. PINK1 import regulation; a fine system to convey mitochondrial stress to the cytosol. BMC Biol 2018; 16:2.

16. Xiao B, Goh J-Y, Xiao L, Xian H, Lim K-L, Liou Y-C. Reactive oxygen species trigger Parkin/PINK1 pathway-dependent mitophagy by inducing mitochondrial recruitment of Parkin. The Journal of biological chemistry 2017; 292:16697-708. 
17. Weidberg H, Shvets E, Elazar Z. Biogenesis and Cargo Selectivity of Autophagosomes. Annual Review of Biochemistry 2011; 80:125-56.

18. Huynh DP, Dy M, Nguyen D, Kiehl TR, Pulst SM. Differential expression and tissue distribution of parkin isoforms during mouse development. Brain Res Dev Brain Res 2001; 130:173-81.

19. McWilliams TG, Prescott AR, Montava-Garriga L, Ball G, Singh F, Barini E, et al. Basal Mitophagy Occurs Independently of PINK1 in Mouse Tissues of High Metabolic Demand. Cell Metab 2018; 27:439-49 e5.

20. Villa E, Proics E, Rubio-Patino C, Obba S, Zunino B, Bossowski JP, et al. ParkinIndependent Mitophagy Controls Chemotherapeutic Response in Cancer Cells. Cell Rep 2017; 20:2846-59.

21. Narendra D, Tanaka A, Suen DF, Youle RJ. Parkin is recruited selectively to impaired mitochondria and promotes their autophagy. J Cell Biol 2008; 183:795-803.

22. Narendra DP, Jin SM, Tanaka A, Suen DF, Gautier CA, Shen J, et al. PINK1 is selectively stabilized on impaired mitochondria to activate Parkin. PLoS Biol 2010; 8:e1000298.

23. Aerts L, Craessaerts K, De Strooper B, Morais VA. PINK1 kinase catalytic activity is regulated by phosphorylation on serines 228 and 402. J Biol Chem 2015; 290:2798-811.

24. Kondapalli C, Kazlauskaite A, Zhang N, Woodroof HI, Campbell DG, Gourlay R, et al. PINK1 is activated by mitochondrial membrane potential depolarization and stimulates Parkin E3 ligase activity by phosphorylating Serine 65. Open Biol 2012; 2:120080.

25. Lee JJ, Sanchez-Martinez A, Zarate AM, Beninca C, Mayor U, Clague MJ, et al. Basal mitophagy is widespread in Drosophila but minimally affected by loss of Pink1 or parkin. J Cell Biol 2018; 217:1613-22.

26. Tang F, Wang B, Li N, Wu Y, Jia J, Suo T, et al. RNF185, a novel mitochondrial ubiquitin E3 ligase, regulates autophagy through interaction with BNIP1. PLoS One 2011; 6:e24367.

27. Di Rita A, Peschiaroli A, D'Acunzo P, Strobbe D, Hu Z, Gruber J, et al. HUWE1 E3 ligase promotes PINK1/PARKIN-independent mitophagy by regulating AMBRA1 activation via IKK $\alpha$. Nature Communications 2018; 9:3755.

28. Rojansky R, Cha MY, Chan DC. Elimination of paternal mitochondria in mouse embryos occurs through autophagic degradation dependent on PARKIN and MUL1. Elife 2016; 5.

29. Yun J, Puri R, Yang H, Lizzio MA, Wu C, Sheng ZH, et al. MUL1 acts in parallel to the PINK1/parkin pathway in regulating mitofusin and compensates for loss of PINK1/parkin. Elife 2014; 3:e01958.

30. Fu M, St-Pierre P, Shankar J, Wang PT, Joshi B, Nabi IR. Regulation of mitophagy by the Gp78 E3 ubiquitin ligase. Mol Biol Cell 2013; 24:1153-62.

31. Orvedahl A, Sumpter R, Jr., Xiao G, Ng A, Zou Z, Tang Y, et al. Image-based genomewide siRNA screen identifies selective autophagy factors. Nature 2011; 480:113-7.

32. Park S, Choi SG, Yoo SM, Son JH, Jung YK. Choline dehydrogenase interacts with SQSTM1/p62 to recruit LC3 and stimulate mitophagy. Autophagy 2014; 10:1906-20. 
33. Szargel R, Shani V, Abd Elghani F, Mekies LN, Liani E, Rott R, et al. The PINK1, synphilin-1 and SIAH-1 complex constitutes a novel mitophagy pathway. Hum Mol Genet 2016; 25:3476-90.

34. Fang S, Ferrone M, Yang C, Jensen JP, Tiwari S, Weissman AM. The tumor autocrine motility factor receptor, gp78, is a ubiquitin protein ligase implicated in degradation from the endoplasmic reticulum. Proc Natl Acad Sci U S A 2001; 98:14422-7.

35. Christianson JC, Olzmann JA, Shaler TA, Sowa ME, Bennett EJ, Richter CM, et al. Defining human ERAD networks through an integrative mapping strategy. Nat Cell Biol 2011; 14:93-105.

36. Zhang T, Kho DH, Wang Y, Harazono Y, Nakajima K, Xie Y, et al. Gp78, an E3 ubiquitin ligase acts as a gatekeeper suppressing nonalcoholic steatohepatitis (NASH) and liver cancer. PLoS One 2015; 10:e0118448.

37. Tsai YC, Mendoza A, Mariano JM, Zhou M, Kostova Z, Chen B, et al. The ubiquitin ligase gp78 promotes sarcoma metastasis by targeting KAI1 for degradation. Nat Med 2007; 13:1504-9. 38. Mukherjee R, Bhattacharya A, Sau A, Basu S, Chakrabarti S, Chakrabarti O. Calmodulin regulates MGRN1-GP78 interaction mediated ubiquitin proteasomal degradation system. FASEB J 2019; 33:1927-45.

39. Mukherjee R, Chakrabarti O. Ubiquitin-mediated regulation of the E3 ligase GP78 by MGRN1 in trans affects mitochondrial homeostasis. J Cell Sci 2016; 129:757-73.

40. Mookherjee D, Das S, Mukherjee R, Bera M, Jana SC, Chakrabarti S, et al. RETREG1/FAM134B mediated autophagosomal degradation of AMFR/GP78 and OPA1 -a dual organellar turnover mechanism. Autophagy 2020.

41. Yang KC, Sathiyaseelan P, Ho C, Gorski SM. Evolution of tools and methods for monitoring autophagic flux in mammalian cells. Biochem Soc Trans 2018; 46:97-110.

42. Kim YY, Um JH, Yoon JH, Kim H, Lee DY, Lee YJ, et al. Assessment of mitophagy in mt-Keima Drosophila revealed an essential role of the PINK1-Parkin pathway in mitophagy induction in vivo. FASEB J 2019; 33:9742-51.

43. Katayama H, Kogure T, Mizushima N, Yoshimori T, Miyawaki A. A sensitive and quantitative technique for detecting autophagic events based on lysosomal delivery. Chem Biol 2011; 18:1042-52.

44. McWilliams TG, Prescott AR, Allen GF, Tamjar J, Munson MJ, Thomson C, et al. mitoQC illuminates mitophagy and mitochondrial architecture in vivo. J Cell Biol 2016; 214:333-45.

45. Klionsky DJ, Abdelmohsen K, Abe A, Abedin MJ, Abeliovich H, Acevedo Arozena A, et al. Guidelines for the use and interpretation of assays for monitoring autophagy (3rd edition). Autophagy 2016; 12:1-222.

46. du Toit A, Hofmeyr JS, Gniadek TJ, Loos B. Measuring autophagosome flux. Autophagy 2018; 14:1060-71.

47. Cardoen B, Wong T, Arabi P, Lee S, Matsubara J, Nabi I, et al. Specht: UnSupervised Probabilistic multiple instance labelling of objects in biomedical images Enables Characterization 
of Transition dynamics in macromolecular structure and disease progression. PLOS One 2021; Submitted.

48. St-Pierre P, Dang T, Joshi B, Nabi IR. Peripheral endoplasmic reticulum localization of the Gp78 ubiquitin ligase activity. J Cell Sci 2012; 125:1727-37.

49. Wang PT, Garcin PO, Fu M, Masoudi M, St-Pierre P, Pante N, et al. Distinct mechanisms controlling rough and smooth endoplasmic reticulum contacts with mitochondria. J Cell Sci 2015; 128:2759-65.

50. Fu M, Li L, Albrecht T, Johnson JD, Kojic LD, Nabi IR. Autocrine motility factor/phosphoglucose isomerase regulates ER stress and cell death through control of ER calcium release. Cell Death Differ 2011; 18:1057-70.

51. Shankar J, Kojic LD, St-Pierre P, Wang PT, Fu M, Joshi B, et al. Raft endocytosis of AMF regulates mitochondrial dynamics through Rac1 signaling and the Gp78 ubiquitin ligase. J Cell Sci 2013; 126:3295-304.

52. St. Pierre P, Nabi IR. The Gp78 ubiquitin ligase: probing endoplasmic reticulum complexity. Protoplasma 2011; Epub ahead of print.

53. Gomez-Sanchez R, Pizarro-Estrella E, Yakhine-Diop SM, Rodriguez-Arribas M, BravoSan Pedro JM, Fuentes JM, et al. Routine Western blot to check autophagic flux: cautions and recommendations. Anal Biochem 2015; 477:13-20.

54. Kimura S, Noda T, Yoshimori T. Dissection of the autophagosome maturation process by a novel reporter protein, tandem fluorescent-tagged LC3. Autophagy 2007; 3:452-60.

55. Katayama H, Yamamoto A, Mizushima N, Yoshimori T, Miyawaki A. GFP-like proteins stably accumulate in lysosomes. Cell Struct Funct 2008; 33:1-12.

56. Barth S, Glick D, Macleod KF. Autophagy: assays and artifacts. The Journal of pathology 2010; 221:117-24.

57. Rubinsztein David C, Shpilka T, Elazar Z. Mechanisms of Autophagosome Biogenesis. Current biology : CB 2012; 22:R29-R34.

58. Kurashige T, Nakajima Y, Shimamura M, Matsuyama M, Yamada M, Nakashima M, et al. Basal Autophagy Deficiency Causes Thyroid Follicular Epithelial Cell Death in Mice. Endocrinology 2019; 160:2085-92.

59. Hara T, Nakamura K, Matsui M, Yamamoto A, Nakahara Y, Suzuki-Migishima R, et al. Suppression of basal autophagy in neural cells causes neurodegenerative disease in mice. Nature 2006; 441:885-9.

60. Chino H, Hatta T, Natsume T, Mizushima N. Intrinsically Disordered Protein TEX264 Mediates ER-phagy. Mol Cell 2019; 74:909-21 e6.

61. Singh R, Kaushik S, Wang Y, Xiang Y, Novak I, Komatsu M, et al. Autophagy regulates lipid metabolism. Nature 2009; 458:1131-5.

62. Delorme-Axford E, Popelka H, Klionsky DJ. TEX264 is a major receptor for mammalian reticulophagy. Autophagy 2019; 15:1677-81.

63. Marcassa E, Kallinos A, Jardine J, Rusilowicz-Jones EV, Martinez A, Kuehl S, et al. Dual role of USP30 in controlling basal pexophagy and mitophagy. EMBO Rep 2018; 19. 
64. Mookherjee D, Das S, Mukherjee R, Bera M, Jana SC, Chakrabarti S, et al. RETREG1/FAM134B mediated autophagosomal degradation of AMFR/GP78 and OPA1 -a dual organellar turnover mechanism. Autophagy 2020:1-24.

65. Musiwaro P, Smith M, Manifava M, Walker SA, Ktistakis NT. Characteristics and requirements of basal autophagy in HEK 293 cells. Autophagy 2013; 9:1407-17.

66. Shirakabe A, Fritzky L, Saito T, Zhai P, Miyamoto S, Gustafsson Å B, et al. Evaluating mitochondrial autophagy in the mouse heart. J Mol Cell Cardiol 2016; 92:134-9.

67. Kirtonia A, Sethi G, Garg M. The multifaceted role of reactive oxygen species in tumorigenesis. Cell Mol Life Sci 2020.

68. Chourasia AH, Tracy K, Frankenberger C, Boland ML, Sharifi MN, Drake LE, et al. Mitophagy defects arising from BNip3 loss promote mammary tumor progression to metastasis. EMBO Rep 2015; 16:1145-63.

69. Tracy K, Dibling BC, Spike BT, Knabb JR, Schumacker P, Macleod KF. BNIP3 is an $\mathrm{RB} / \mathrm{E} 2 \mathrm{~F}$ target gene required for hypoxia-induced autophagy. Mol Cell Biol 2007; 27:6229-42.

70. Joshi B, Li L, Nabi IR. A role for KAI1 in promotion of cell proliferation and mammary gland hyperplasia by the gp78 ubiquitin ligase. J Biol Chem 2010; 285:8830-9.

71. Joshi B, Strugnell SS, Goetz JG, Kojic LD, Cox ME, Griffith OL, et al. Phosphorylated caveolin-1 regulates Rho/ROCK-dependent focal adhesion dynamics and tumor cell migration and invasion. Cancer Res 2008; 68:8210-20.

72. Cardoen B, Ben Yedder H, Sharma A, Chou KC, Nabi IR, Hamarneh G. ERGO: Efficient Recurrent Graph Optimized Density Estimation in Single Molecule Localization Microscopy. Trans Med Imaging 2019; Epub ahead of print. 


\section{FIGURE LEGENDS}

Figure 1: CRISPR/Cas9 knockout of Gp78 in HT-1080 prevents damage-induced mitophagy. (A) Schematic showing Exon 1 region of Gp78 gene on chromosome 16 and location of gRNA1 targeting the start codon, deleting ATG ( $\Delta \mathrm{G}$ or inserting extra $\mathrm{T})$, and of gRNA2, inserting an extra $\mathrm{T}$ at amino acid 16 causing frameshift and termination. Western blot for Gp78 is shown for wildtype HT-1080 cells and all six gRNA1 and gRNA2 Gp78 knockout CRISPR clones with $\beta$-actin as a loading control. (B) Wild-type HT-1080 cells and the six Gp78 knockout CRISPR clones were incubated with either DMSO or $10 \mu \mathrm{M}$ CCCP for 24 hours then fixed and labeled for mitochondrial ATPB and imaged by 3D spinning disk confocal microscopy. Representative images of HT-1080 and a CRISPR clone including magnification of boxed region are shown. Quantification of total mitochondrial volume is shown in the bar graph $(\mathrm{n}=3 ; *, \mathrm{p}<0.05 ; * *, \mathrm{p}<0.01 ; * * *, \mathrm{p}<0.001$ relative to DMSO or CCCP treated HT-1080 cells, respectively; \pm SEM; Scale Bars: $5 \mu \mathrm{m}$; $1 \mu \mathrm{m}$ for zooms).

Figure 2: Gp78 induces basal mitophagy in HT-1080 cells. (A) Wild-type HT-1080 cells and the g1-4 and g2-41 Gp78 knockout CRISPR clones were transfected with non-specific siCTL or siATG5 and western blotted for ATG5, mitochondrial ATPB and $\beta$-actin. Densitometry quantification of ATBP relative to $\beta$-actin is shown in the bar graph. (t-test, $\mathrm{n}=3, * * \mathrm{p}<0.001$, \pm SEM). (B) Wild-type HT-1080 cells and the g1-4 and g2-41 Gp78 knockout CRISPR clones transfected with non-specific siCTL or siATG5 were incubated with either DMSO or $10 \mu \mathrm{M} \mathrm{CCCP}$ for 24 hours and then fixed and labeled for mitochondrial ATPB and imaged by 3D spinning disk confocal microscopy. Quantification of total mitochondrial volume is shown in the bar graph. $(\mathrm{n}=3, *, \mathrm{p}<0.05 ; * *, \mathrm{p}<0.01 ; \pm \mathrm{SEM})$.

Figure 3: Flux of autophagy protein LC3B-II in wild-type HT-1080 and Gp78 knockout cell lines. Western blot analysis and probing of LC3B-II in HT-1080 cells and g1-4 and g2-41 Gp78 knockout CRISPR/Cas clones after 4 hours treatment with either DMSO, CCCP or starvation in presence of $100 \mathrm{nM}$ of BafA1 for 0, 30, 60 and 120 minutes. DMSO or serum-starved cells show increased and accumulated LC3B-II with BafA1 in time-dependent manner. The slope of LC3BII band density quantified relative to $\beta$-actin show significant differences between the three cell lines; LC3B-II flux in CCCP treatment of HT-1080 cells but not in the Gp78 knockout clones can be $\operatorname{seen}(\mathrm{n}=4, *, \mathrm{p}<0.05 ; * * ; \pm \mathrm{SEM})$.

Figure 4: SPECHT spot detection detects LC3 puncta and basal autophagic flux. (A) Raw confocal images, Laplacian transforms and SPECHT ( $\mathrm{z}=1.75)$ spot detection of GFP (green) and RFP (red) tfLC3 signal as well as overlay of GFP-RFP-positive tfLC3 puncta (yellow) with a mask of MitoView mitochondrial labeling (blue) are shown. (B) A parameter sensitivity study testing various z-values to determine the significance levels between overlapping GFP-mRFP tfLC3 spots in HT-1080 cells treated with either DMSO or with CCCP and BafA1. Y-axis denotes the mean 
number of detected spots. (C) A parameter sensitivity study testing various z-values and their effect on the mean of number of overlapping GFP-mRFP tfLC3 spots per cell with mitochondria, within or equal to the 5-pixel unit resolution limit. Bonferroni correction with $\mathrm{m}=252$ is applied to correct for multiple hypothesis testing. The Kruskal non-parametric test is applied to test if the samples originate from the same distribution. (D) Bar graphs show the number of overlapping GFP-LC3 and mRFP-LC3 spots per HT-1080 cell treated with DMSO or CCCP \pm BafA1, total and in association with mitochondria, per cell (Mean \pm SEM; 10-20 cells/experiment; $\mathrm{n}=6, * * * \mathrm{p}<$ $0.001, * * * * \mathrm{p}<0.0001)$. Scale bar, $10 \mu \mathrm{m}$.

Figure 5: Gp78 knockout in HT-1080 cells results in impaired autophagic flux. (A) Masks of GFP-mRFP-positive tfLC3 puncta (yellow) and mitochondria (blue) in live HT-1080 and g2-41 cells stably transfected with tfLC3 and labelled with the mitochondrial dye MitoTracker Deep Red FM. Live cells were either treated with $100 \mathrm{nM}$ BafA1 and imaged at the indicated time points. Bar graphs show the number of GFP-mRFP-positive tfLC3 puncta, total and in association with mitochondria, per cell (Mean $\pm \mathrm{SEM} ; \mathrm{n}=3,{ }^{*} \mathrm{p}<0.05, * * \mathrm{p}<0.01$ ). Scale bar, $10 \mu \mathrm{m}$. (B) GFP-mRFPpositive tfLC3 puncta (yellow) and mitochondria (blue) masks of HT-1080 and g2-41 Gp78 KO cells stably transfected with tfLC3 and labelled with mitochondrial dye MitoTracker Deep Red FM transfected with either control of siATG5 siRNA and treated with $100 \mathrm{nM}$ BafA1. Bar graphs show the number of GFP-mRFP-positive tfLC3 puncta, total and in association with mitochondria, per cell (Mean \pm SEM; $n=3, * p<0.05, * * p<0.01$ ). (Mean \pm SEM; $n=3, * p<0.05, * * * p<0.001)$. Scale bar, $10 \mu \mathrm{m}$.

Figure 6. Basal mitophagy maintains healthy mitochondria and limits ROS production in HT-1080 cells. HT-1080 and Gp78 KO g2-42 HT-1080 cells were transfected with siCTL (black bars) or siATG5 (white bars) and labeled with either the mitochondrial potential reporter MitoView 633 (A) or the mitochondrial ROS probe MitoSox (B) and (red) for 30 minutes. Live confocal images are shown and MitoView and MitoSox intensity and area quantified per cell. (Scale Bar: $10 \mu \mathrm{m} ; \mathrm{n}=3 ; * * \mathrm{p}<0.01 ; * * * * \mathrm{p}<0.001$ ).

Figure 7. Time lapse imaging of tfLC3 in HT-1080 cells shows autophagosome association with low potential mitochondria. (A) Z-stacks (7 images; $500 \mathrm{~nm}$ spacing; 14 cells) of HT-1080 cells expressing tfLC3 and labeled with MitoView were acquired by spinning disk confocal every minute over 40 minutes and processed by SPECHT to reveal GFP-mRFP-positive tfLC3 puncta and mitochondria. A representative image of a single Z-section shows SPECHT processing of tfLC3 and mitochondria signals (Scale bar: $10 \mu \mathrm{m}$ ). ROI shows overlap of autophagosomes with lower potential mitochondria (Scale bar: $2 \mu \mathrm{m}$ ). Average intensity of MitoView positive pixels overlapping GFP-mRFP-positive tfLC3 puncta was quantified relative to the average of all MitoView positive pixels in the adjacent mitochondrial segment or in all mitochondrial segments in the cell. For each GFP-mRFP-positive tfLC3 puncta in any of the 7 Z-slices, the ratio of overlapping mitochondria pixels relative to adjacent mitochondria segment and all mitochondria 
segments in the cell is shown per cell as a boxplot $(<1$ : fainter than average; $>1$ brighter than average). (B) Image series from time lapse movies, as described in (A), acquired every 10 seconds are shown (see Supplemental video 1). Boxplots are as described in (A). (Scale Bar: $2 \mu \mathrm{m} ; \mathrm{n}=4$ ). 
A

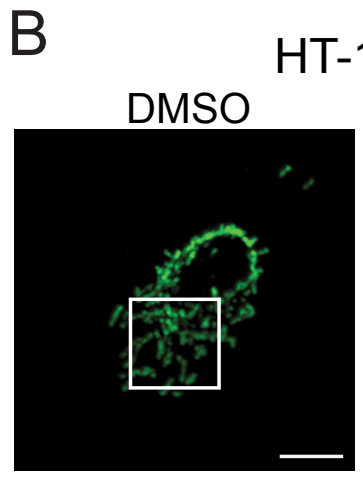

hGp78 Exon1 (chromosome 16)
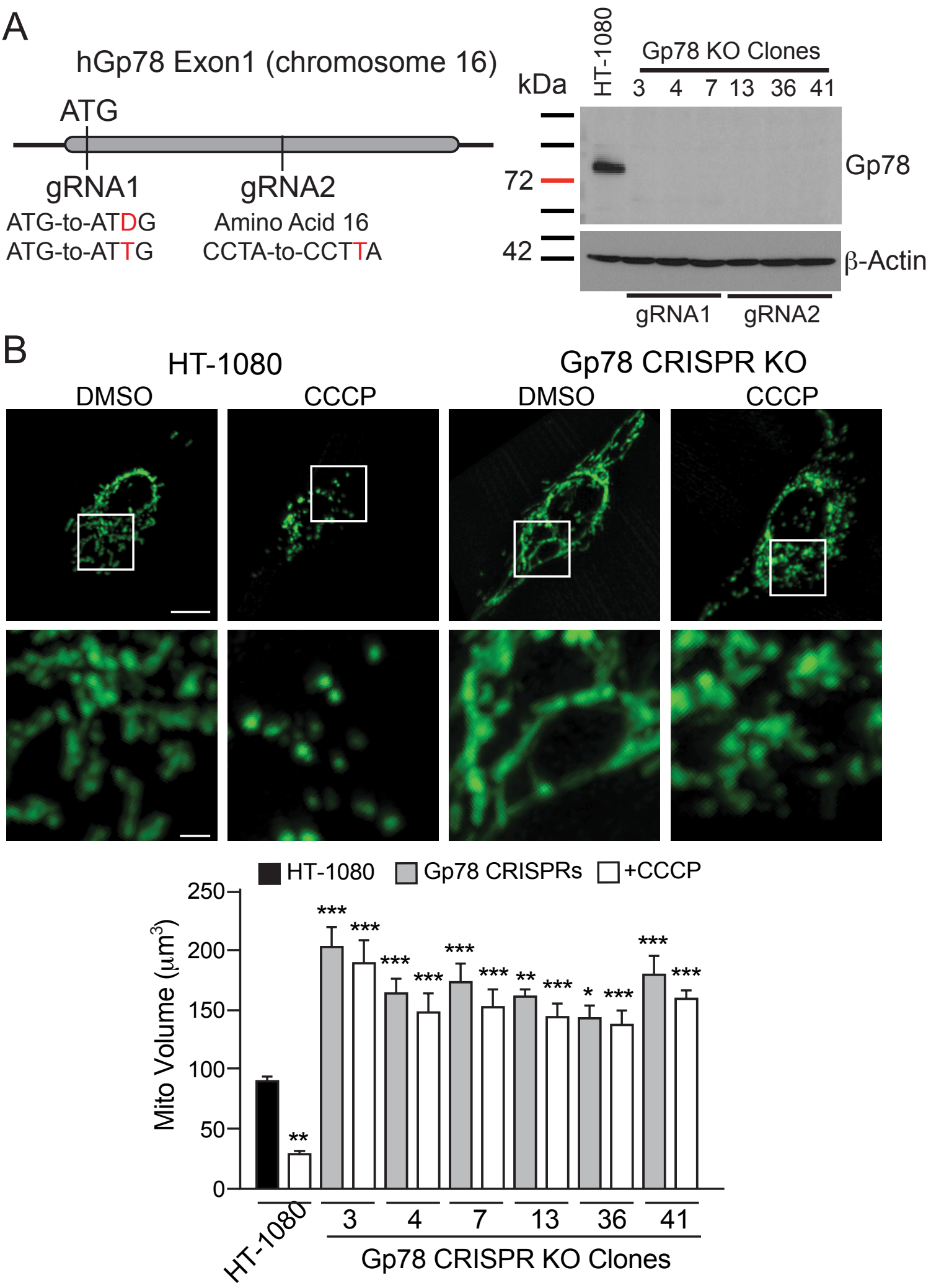

Figure 1 
A
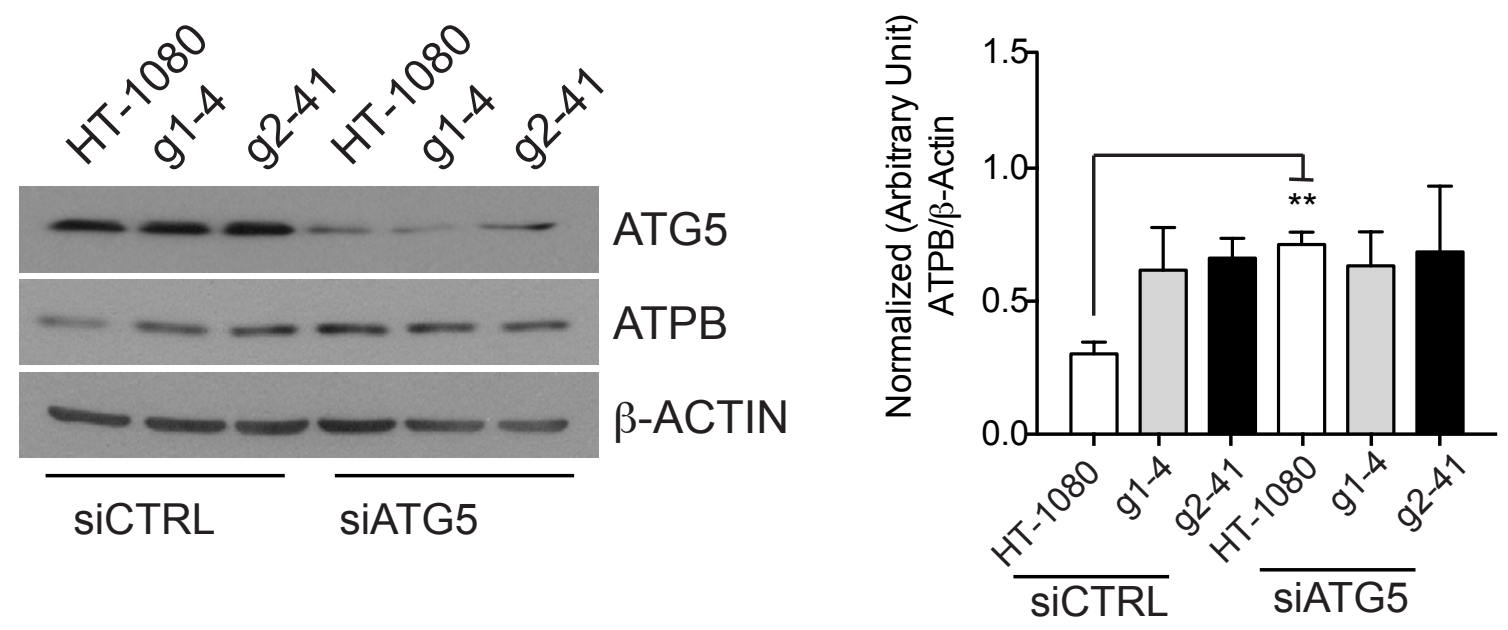

B
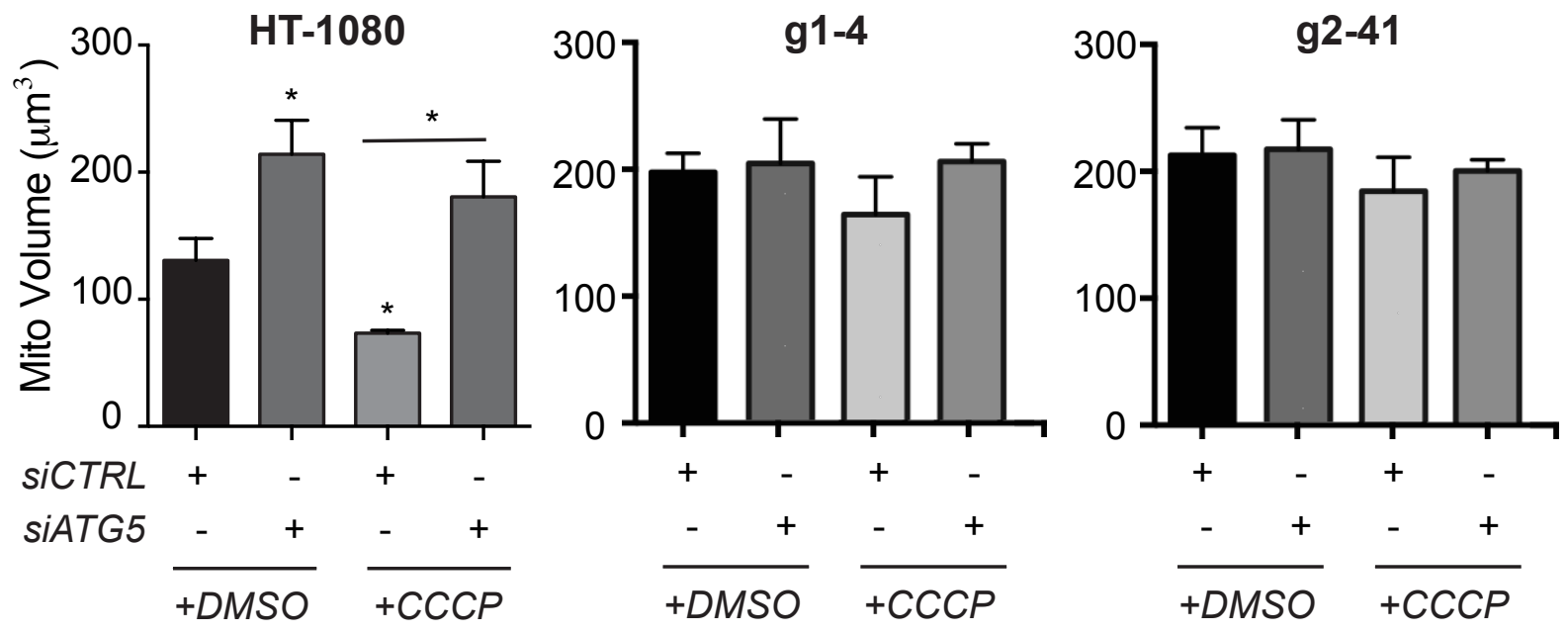

Figure 2 
bioRxiv preprint doi: https://doi.org/10.1101/2021.09.17.460825; this version posted September 17, 2021. The copyright holder for this preprint (which was not certified by peer review) is the author/funder. All rights reserved. No reuse allowed without permission.
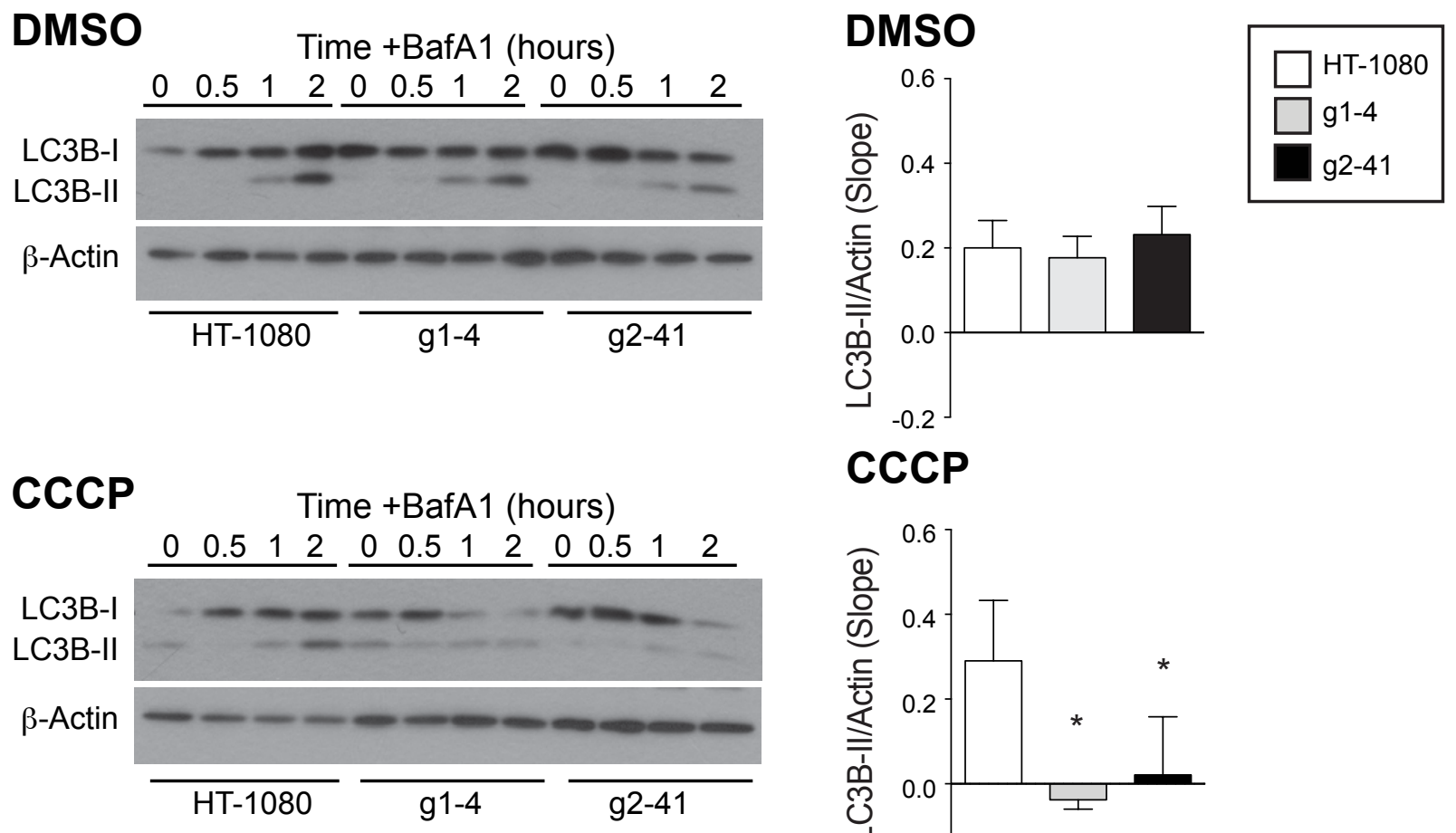

\section{CCCP}

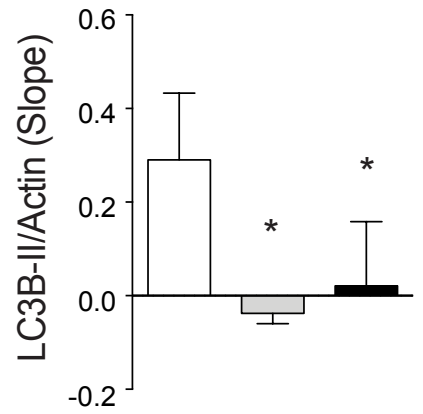

\section{Starvation}

$$
\text { Time +BafA1 (hours) }
$$

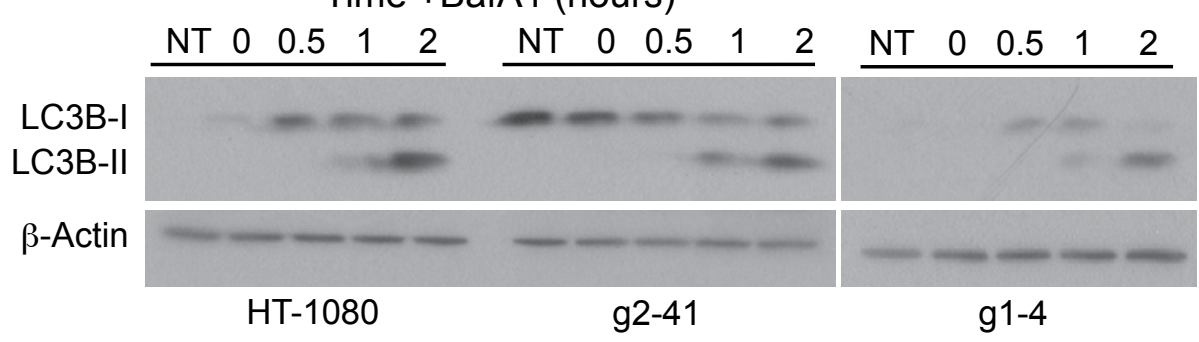

\section{Starvation}

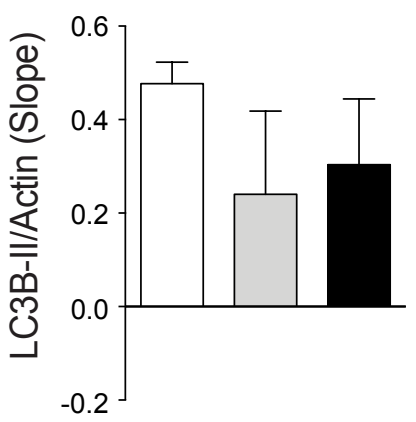


A

GFP-LC3/mRFP-LC3
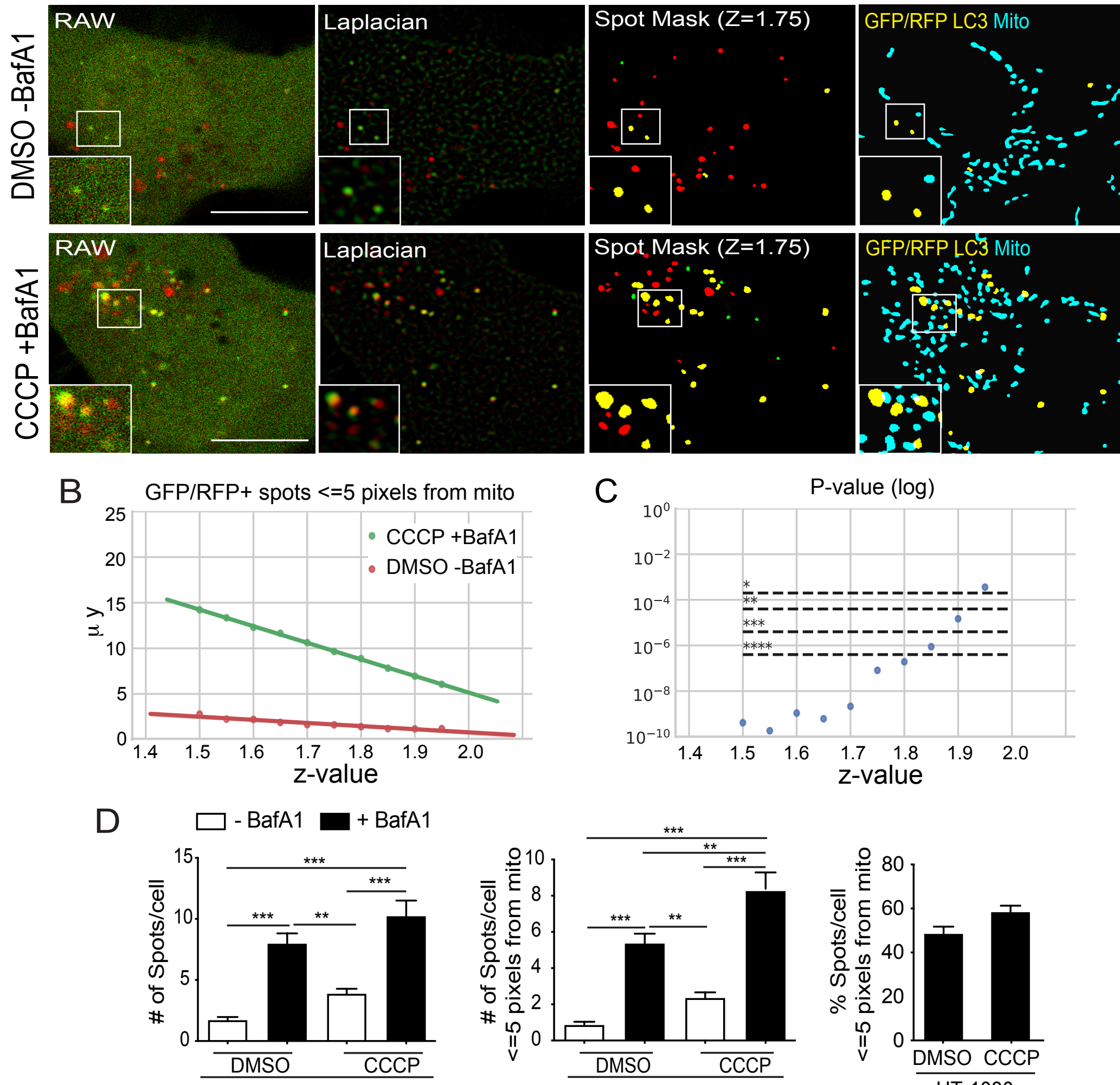

HT-1080

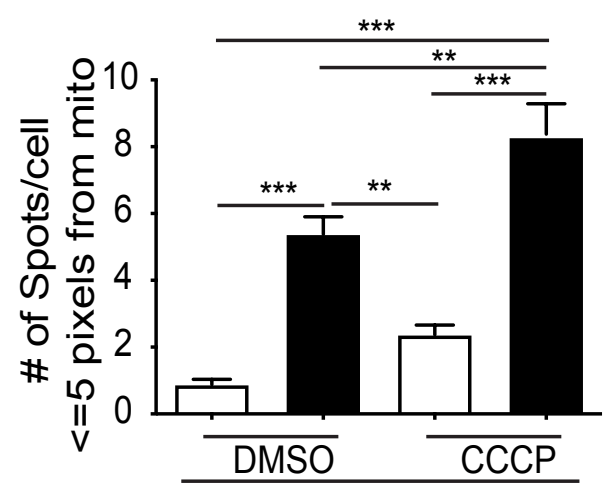

HT-1080

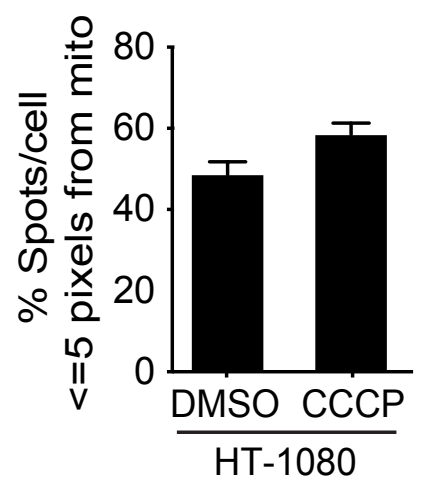

Figure 4 


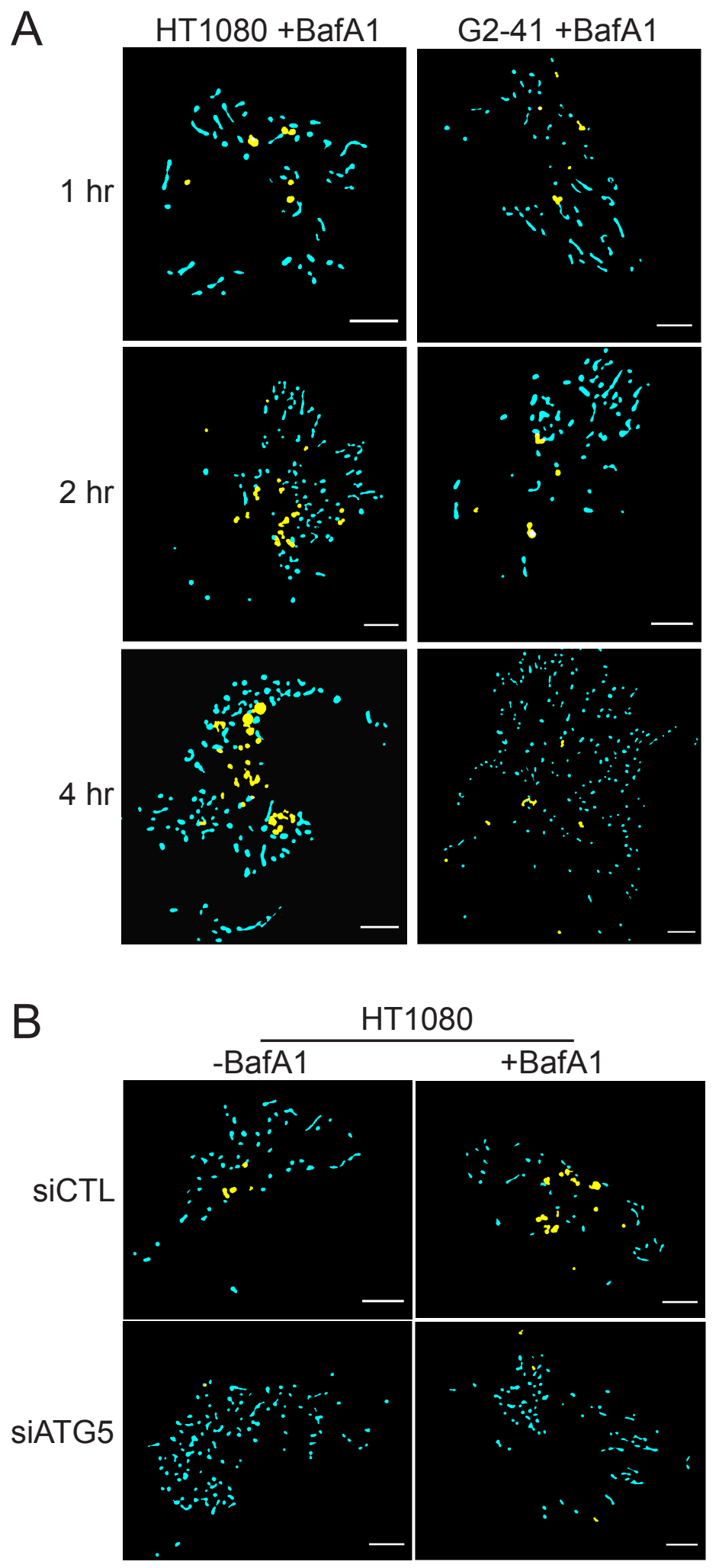

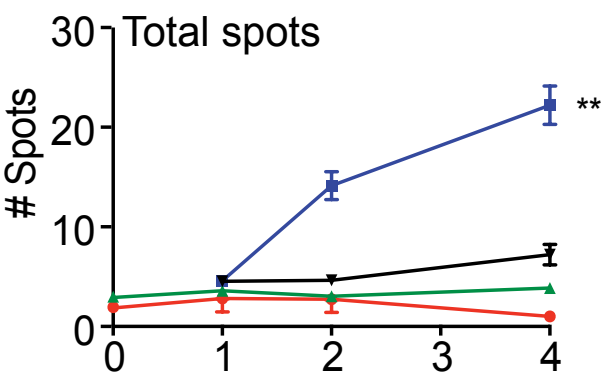

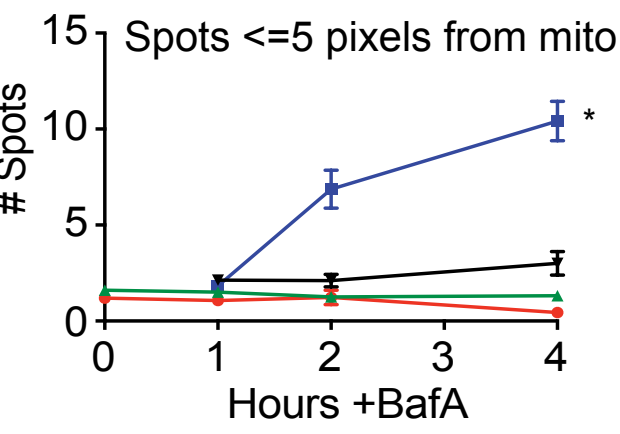

\begin{tabular}{ll}
\hline HT-1080 & $g 2-41$ \\
- -BafA1 & $\neq-$-BafA1 \\
- +BafA1 & - +BafA1
\end{tabular}
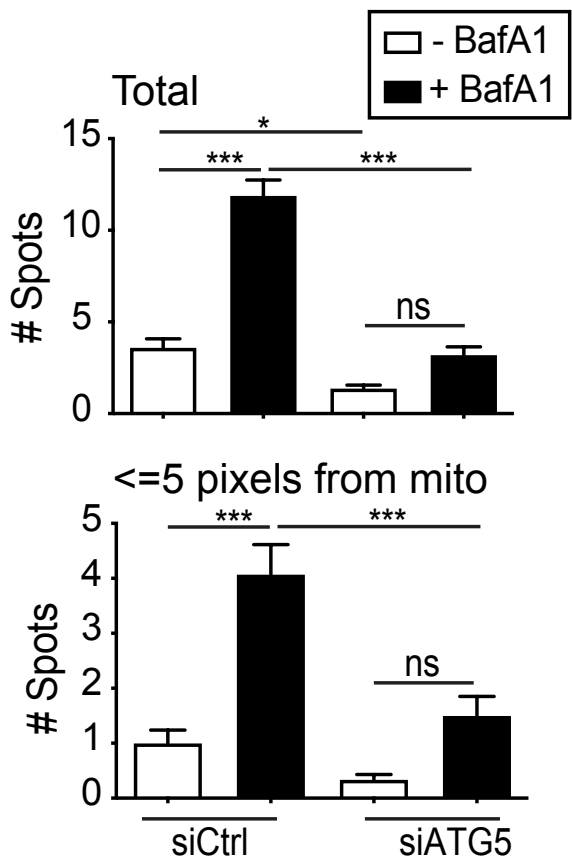
A
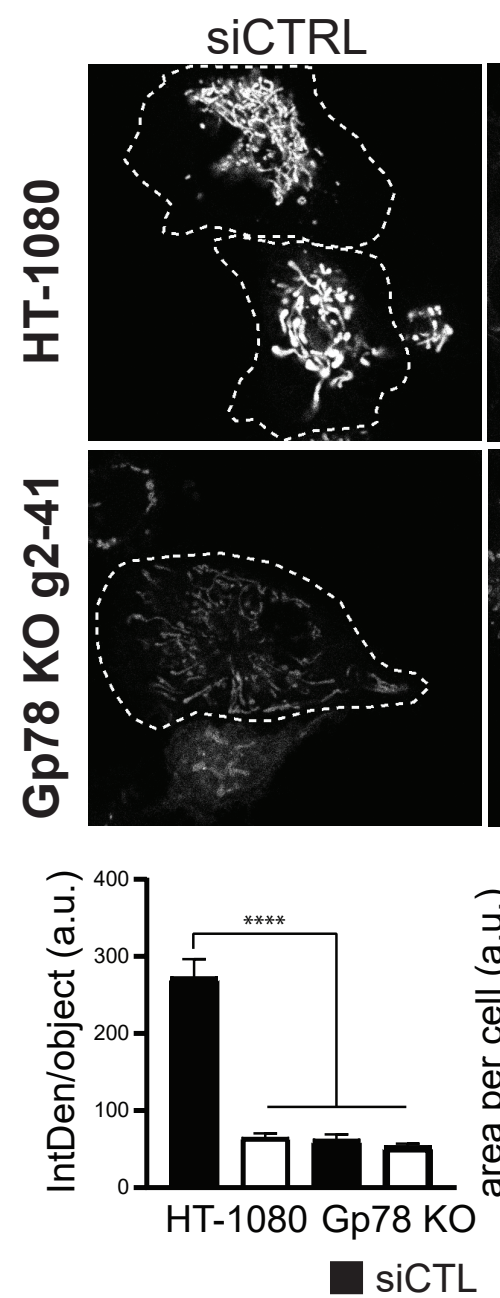

MitoView 633
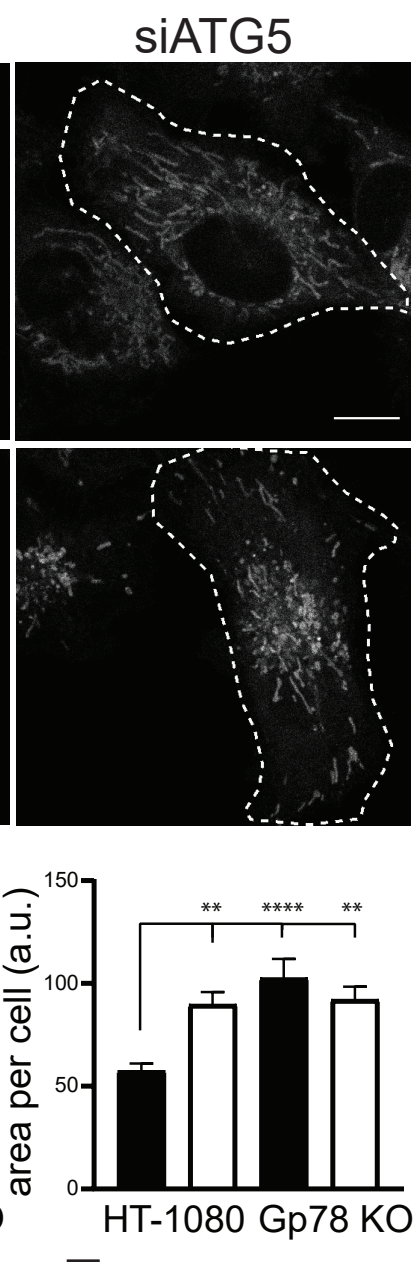

B
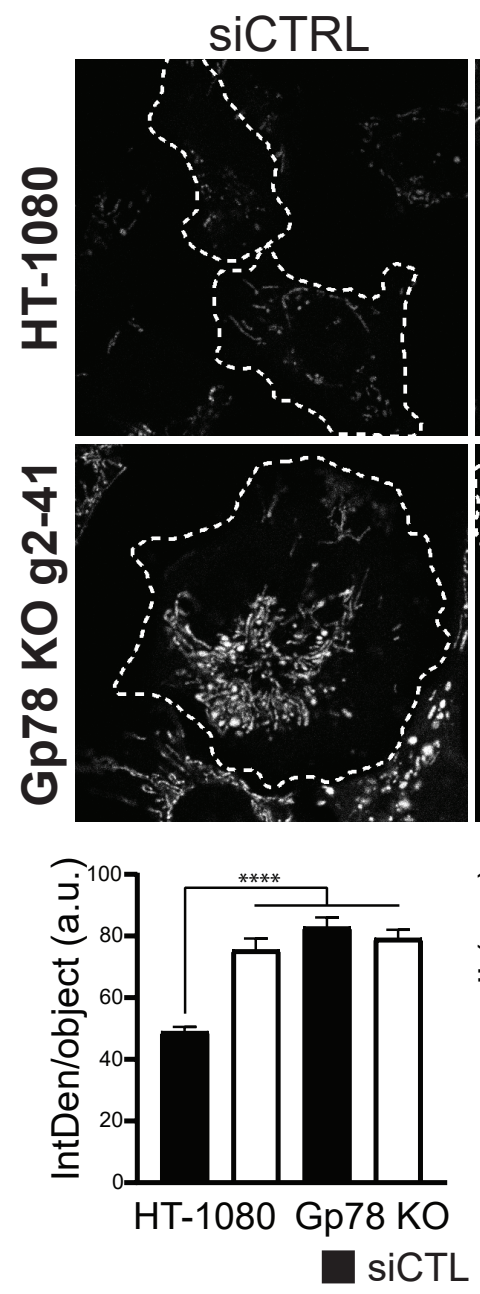

MitosoX
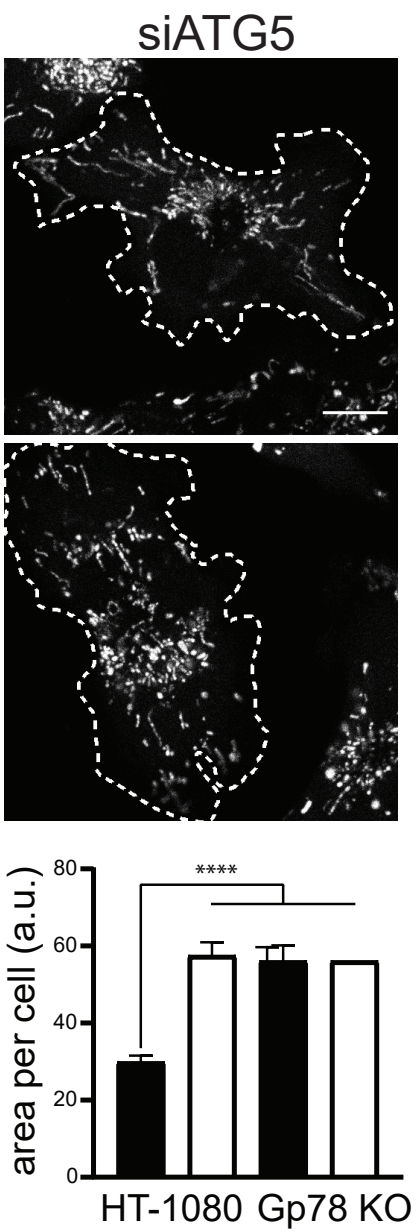

HT-1080 Gp78 KO siATG5 
bioRxiv preprint doi: https://doi.org/10.1101/2021.09.17.460825; this version posted September 17, 2021. The copyright holder for this preprint (which was not certified by peer review) is the author/funder. All rights reserved. No reuse allowed without permeissilios,
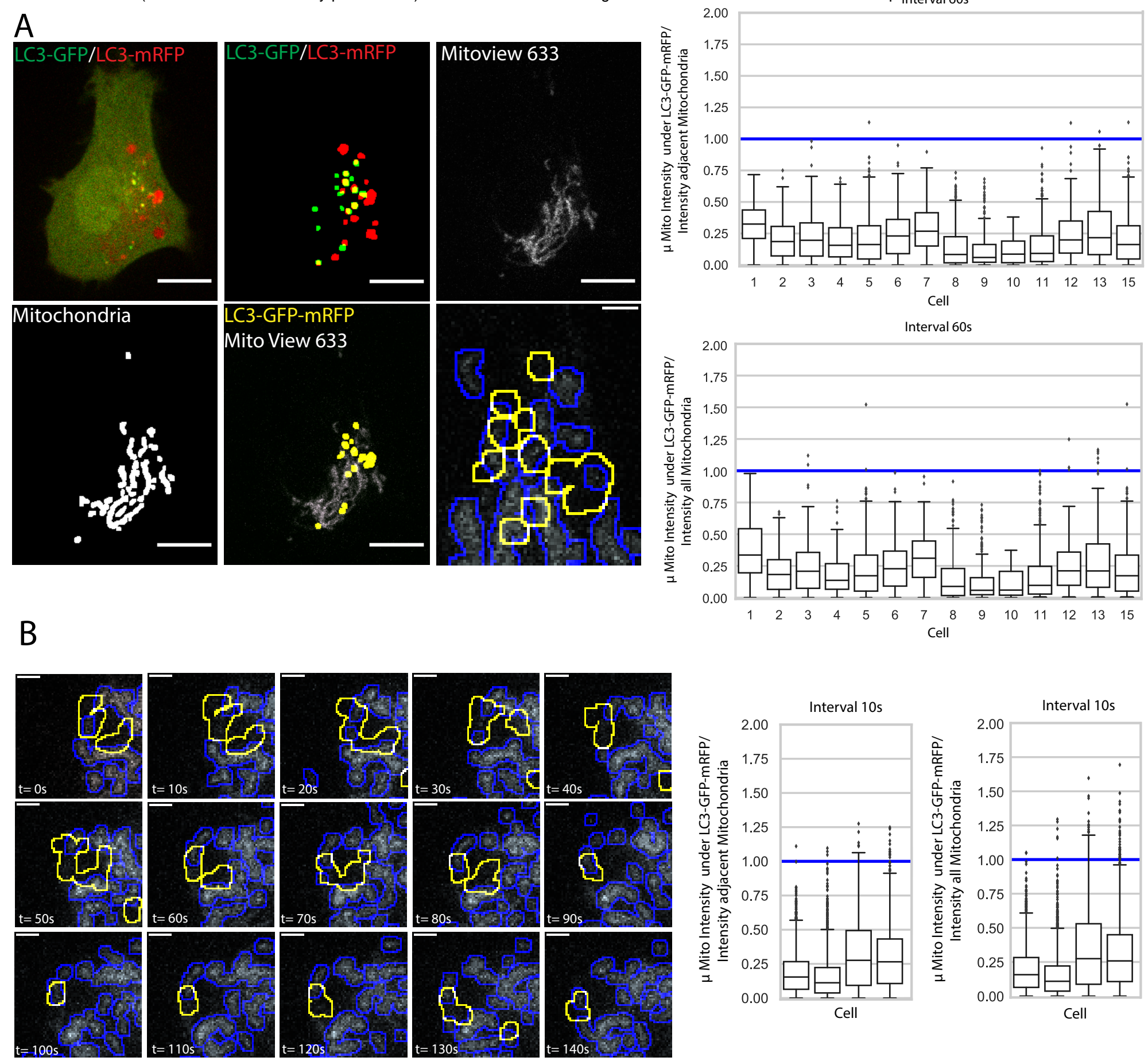

Figure 7 